\title{
L'organisation des temps dans les activités d'entraînement : des inventions locales dans un cadre contraignant
}

\author{
Anne-Claire Macquet \\ Collaborateur scientifique : P. Fleurance
}

Travail effectué par

Laboratoire de psychologie et d'ergonomie du sport, INSEP, Paris

En collaboration avec

Groupe de recherche sur l'analyse des pratiques professionnelles et l'ingénierie de formation, INSEP, Paris

Téléchargeable sur le site : http://sciences.campus-insep.com

Institut National du Sport et de l'Education Physique

11, Avenue du Tremblay - 75012 Paris

0141744100 -www.insep.fr
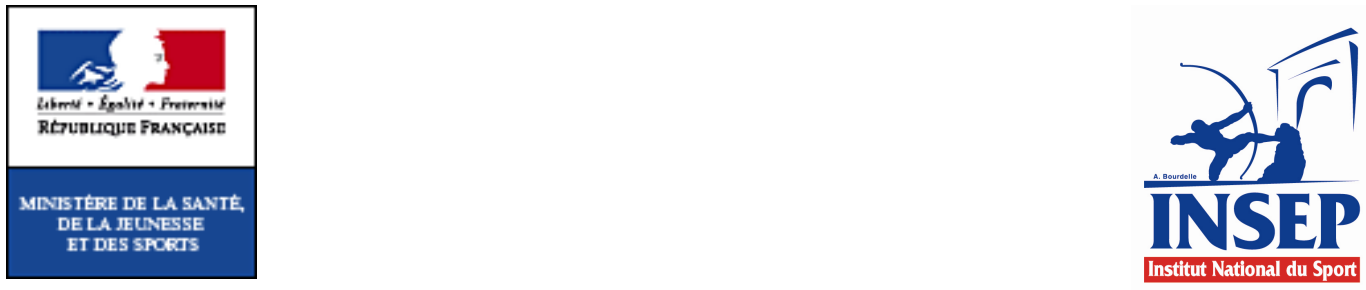


\section{REMERCIEMENTS}

Cette étude a été soutenue financièrement par le Ministère de la Santé et de la Jeunesse et des Sports.

Les auteurs de ce rapport remercient sincèrement les entraîneurs, pour leur participation active à l'étude.

Les résultats de cette étude ont été présentés lors des Entretiens de l'INSEP, intitulés : « Interroger les entraîneurs au travail. Revisiter les conceptions qui organisent l'entraînement pour revisiter le métier d'entraîneur » (11 et 12 octobre 2007).

Ces résultats seront publiés prochainement dans un ouvrage collectif.

Macquet, A.-C. (sous presse). L'organisation des temps dans les activités d'entraînement : des inventions locales dans un cadre contraignant. In P. Fleurance, \& S. Perez (Eds), Interroger les entraîneurs au travail. Revisiter les conceptions qui organisent l'entraînement pour revisiter le métier d'entraîneur (pp. 289-319). Paris : Editions INSEP. 


\section{Sommaire}

$\begin{array}{ll}\text { Introduction } & 5\end{array}$

$\begin{array}{ll}\text { 1. Revue de littérature } & 7\end{array}$

$\begin{array}{ll}\text { 1.1. Une organisation rationnelle du temps } & 7\end{array}$

1.2. D'une organisation collective à une organisation individualisée du temps de l'entraînement $\quad 8$

1.3. Des micro-organisations temporelles locales au sein de l'entraînement $\quad 9$

1.4. Des organisations temporelles dans une structure institutionnelle $\quad 9$

1.5. Deux conceptions distinctes du temps à l'origine de modes

d'organisation particuliers

1.6. Les buts de l'étude $\quad 10$

2. Méthode 11

$\begin{array}{ll}\text { 2.1. Les participants } & 11\end{array}$

2.2. Le recueil des données 11

2.3. Le traitement des données $\quad 14$

3. Résultats 15

3.1. Le processus de génération des organisations temporelles 15

$\begin{array}{ll}3.1 .1 \text {. Un processus circulaire } & 15\end{array}$

3.1.2. Trois paramètres en interaction $\quad 16$

$\begin{array}{ll}3.2 .1 .1 . \text { Les compétitions } & 16\end{array}$

$\begin{array}{ll}\text { 3.2.1.2. Les projets } & 17\end{array}$

3.2.1.3. L'entraînement 19

3.2. Les organisations temporelles : des inventions locales issues de compromis $\quad 22$

3.2.1. Sauvegarder du temps pour maintenir une préparation de qualité

tout en répondant aux attentes des athlètes $\quad 22$

3.2.2. Privilégier les compétitions sélectives tout en maintenant une durée
de préparation optimale entre deux compétitions

3.2.3. Favoriser le retour dans les familles tout en préservant du temps pour préparer les compétitions 24

3.2.4. Individualiser l'entraînement tout en organisant le travail pour le groupe 24 
3.2.5. Optimiser les temps d'entraînement tout en respectant les temps de l'institution

3.2.6. Libérer des temps pour se reposer tout en occupant ces temps pour ne pas s'ennuyer

3.3. Les organisations temporelles : des inventions locales propres à chaque sport $\quad 26$

$\begin{array}{ll}\text { 3.3.1. Deux types de bornage des temps } & 26\end{array}$

$\begin{array}{ll}\text { 3.3.2. Trois organisations typiques au cours de la saison } & 27\end{array}$

$\begin{array}{ll}\text { 3.3.2.1. Une organisation en deux périodes larges pour le groupe } & 27\end{array}$

3.3.2.2. Une organisation en trois périodes larges pour le groupe 28

3.3.2.3. Une organisation en différentes périodes pour chacun des athlètes $\quad 28$

$\begin{array}{ll}\text { 3.3.3. Des relations entre les périodes } & 29\end{array}$

3.3.4. La confrontation entre des temps prévus et des temps réels 30

4. Discussion $\quad 32$

4.1. Un processus circulaire de génération des organisations temporelles révélant une vision holistique des activités d'entraînement

4.2. Les organisations temporelles : des inventions locales finalisées par la réussite dans un cadre contraignant

4.3. Des organisations temporelle typiques liées à des temporalités variées

35

4.4. Des perspectives pour la formation des entraîneurs

Conclusion 


\section{Introduction}

"C'est 10 fois mieux quand on est en stage parce qu'on est complètement concentré sur ce qu'on a à faire, on a les athlètes sous la main, eux aussi d'ailleurs sont complètement concentrés sur ce qu'ils font. C'est simple quand on est en stage, il y a le judo, l'entraînement, les objectifs liés à ça et la vie quotidienne qui passe après presque, c'est d'abord centré sur l'entraînement, c'est pour ça que c'est payant. Parce que quand on est ici à l'INSEP ils ont d'abord leurs vies, leurs quotidiens et ensuite dans leurs quotidiens il y a l'entraînement. »

"Cette athlète a commencé des études pour préparer son insertion professionnelle. Ca a complètement changé son rythme de vie. Auparavant, elle était vraiment joueuse [...] professionnelle : elle s'entraînait et se reposait. Là, on a à gérer le facteur fatigue, ce qui fait qu'on a changé l'organisation de la journée, de la semaine. »

L'entraînement ne semble pas pouvoir être isolé de la vie des athlètes de haut niveau, il en fait partie intégrante. La vie des athlètes ne se limite pas à l'entraînement. Si le projet sportif est au centre de leurs préoccupations, les athlètes sont amenés à doubler ce projet pour envisager leur avenir professionnel, au delà de leur carrière sportive. Ce doublement du projet apparaît comme une particularité française, qui amène à des organisations particulières du temps, au cours de la journée, de la semaine, de l'année. Dans ces conditions, l'organisation du temps dans les activités d'entraînement ne peut se réduire à la principale prise en compte du temps de l'entraînement. Les temps consacrés aux autres activités ne peuvent être occultés. Certaines activités peuvent avoir des incidences sur d'autres: elles peuvent induire de la fatigue ou ne pas permettre de se reposer, elles peuvent être à l'origine de préoccupations différentes pour les athlètes, qui peuvent alors être amenés à être moins focalisés sur le travail sportif, lors des séances d'entraînement. Les interactions entre ces activités conduisent à reconsidérer le temps dans l'entraînement. D'un temps essentiellement centré sur l'entraînement (e.g., Platonov, 1988), on passe à des temps focalisés sur les activités d'entraînement, de formation, les activités professionnelles, le repos... Un changement de centre s'opère. Le temps de l'entraînement n'est plus le seul pris en compte, il interagit avec d'autres temps, qui sont liés aux activités des athlètes et des entraîneurs dans des espaces particuliers. Ces temps apparaissent co-déterminés. Le temps n'apparaît plus unique, mais plutôt multiple. Il ne semble plus continu, mais discontinu: des temps se succèdent, s'imbriquent, se superposent parfois, générant ainsi des concurrences, comme c'est le cas lors des périodes d'examen, de stages sportifs organisés pendant les périodes scolaires ou universitaires...

Les activités d'entraînement sont organisées dans un cadre local, dont les circonstances sont sans cesse renouvelées. Ce cadre renvoie à la fois aux structures dans laquelle se déploient les activités d'entraînement et aux agents intervenant dans ce cadre. En France, la structure consiste le plus souvent en un pôle d'entraînement ou un club. De façon schématique, les structures de pôles prévoient des temps pour les différentes activités; elles contribuent à l'organisation de ces activités, tandis que les structures de clubs se centrent principalement sur l'entraînement. La majorité des médaillés olympiques est issue d'une structure de pôles 
particulière : l'Institut National des Sports et de l'Education Physique (INSEP). L'INSEP met en place les conditions de la réussite sportive tout en permettant aux athlètes de préparer leur reconversion, en favorisant une formation scolaire, universitaire ou professionnelle. L'institut structure les temps pour permettre la réalisation des activités relevant des différents projets. Dans les pôles sportifs, s'organisent les temps liés aux activités d'entraînement. L'organisation des temps dans les activités d'entraînement est générée dans un cadre institutionnel commun à l'ensemble des sports. Cette organisation des temps s'inscrit également dans le cadre de fédérations sportives nationales et internationales, qui déterminent un ensemble d'éléments structurant l'organisation locale des temps dans le pôle. Ces éléments renvoient principalement aux compétitions (date, enjeu...). Ils varient en fonction des fédérations concernées. Les agents déploient leurs activités dans ces cadres institutionnels. Ces activités sont finalisées par un projet sportif, doublé par un projet scolaire, universitaire ou professionnel, et parfois familial. Leurs préoccupations et leur disponibilité évoluent de façon dynamique. Elles sont en partie déterminées par les priorités accordées aux différents projets dans l'espace et dans le temps.

C'est dans ce contexte un peu particulier, qu'il apparaît intéressant d'étudier l'organisation des temps dans les activités d'entraînement. Cette organisation semble déterminée à la fois par le projet sportif des athlètes, mais aussi par le doublement de ce projet et par les structures au sein desquelles les activités d'entraînement se déploient. La structure INSEP prévoit une organisation des temps au cours de la journée, de la semaine et de l'année, pour l'ensemble des athlètes et des entraîneurs. La structure pôle s'inscrit dans la précédente. Des temps focalisés sur les activités d'entraînement y sont organisés pour chacun des athlètes, en fonction de leurs disponibilités aux différents moments de la journée, de la semaine, de l'année. Ces disponibilités peuvent évoluer dans le temps. Ces organisations sont souvent locales et contingentes au contexte. Il apparaît intéressant de s'interroger sur l'organisation locale et circonstancielle des temps dans les activités d'entraînement, afin d'examiner la portée heuristique des modèles de planification de l'entraînement. 


\section{Revue de littérature}

\subsection{Une organisation rationnelle du temps}

Les activités d'entraînement sont souvent considérées comme des activités de planification et de régulation finalisées par la réalisation de performances en compétition (Platonov, 1988; Weineck, 1986 ; Pradet, 1996). Les planifications s'appuient essentiellement sur des connaissances issues de la physiologie, de la biomécanique, de l'activité sportive considérée et de la psychologie du sport. Elles concernent des durées différentes qui vont le plus souvent d'une planification pluriannuelle à la planification de la séance. Elles visent à découper le temps en périodes plus ou moins longues, finalisées par une préparation progressive aux compétitions (e.g., périodes de préparation générale, spécifique ou de compétition, Platonov, 1988). Les planifications sont adaptées au groupe d'entraînement puis aux athlètes. Des progressions dans la charge et le volume d'entraînement, dans les apprentissages techniques et tactiques sont ensuite déterminées. Les planifications sont le plus souvent conçues pour des cycles de compétitions éloignés les uns des autres (e.g., périodisation simple ou double, Weineck, 1986). Or la multiplication des compétitions internationales et l'engagement différencié des athlètes aux compétitions variées semblent bousculer ce type de périodisation, conçue le plus souvent pour le groupe d'entraînement. Ces phénomènes amènent à la fois à une sélection des épreuves pour chacun des athlètes et à une périodisation « à la carte ». D'un découpage du temps pour l'ensemble du groupe d'entraînement, on s'achemine vers des découpages multiples en fonction du niveau des athlètes, du nombre de compétitions, de leur emplacement au cours de la saison, de leurs enjeux pour les sportifs.

Par ailleurs, les planifications qui apparaissent dans la littérature sur l'entraînement sont conçues sous forme algorithmique. Elles associent des conditions particulières, des actions spécifiques et des effets attendus. Ainsi, l'augmentation progressive du volume et de la charge d'entraînement en fonction du niveau des athlètes et des effets attendus à un moment particulier permet de faire reculer l'apparition de la fatigue. Cette conception algorithmique de l'entraînement s'inscrit dans une démarche rationnelle, dans laquelle le planificateur tente de contrôler l'ensemble des variables liées à l'entraînement. Les planifications ainsi construites visent à optimiser la préparation de l'athlète et à écarter les imprévus liés à cette préparation: blessure, fatigue importante et durable, épuisement psychologique. Les planifications sont évaluées et régulées en fonction des effets observés. Ces planifications sont conçues à partir de connaissances scientifiques sur l'entraînement : physiologie de l'effort, biomécanique, psychologie... Cette conception de l'entraînement s'appuie sur une épistémologie de la « rationalité technique » (Schön, 1983). L'entraînement est fondé sur des lois spécifiques qui prévoient le développement de la performance sportive. Les actions d'entraînement sont prescrites à travers des planifications préalables. Ces planifications s'appuient sur un temps linéaire, unique et continu: les périodes et sous périodes se succèdent et sont bornées dans le temps. Ce bornage répond à une durée établie au cours de l'activité de planification. 


\subsection{D'une organisation collective à une organisation individualisée du temps de l'entraînement}

Dans une étude menée sur le métier d'entraîneur, Salmela (1996) a envisagé le temps à travers la planification de la saison et la fixation d'objectifs. Les planifications réalisées par les entraîneurs s'appuyaient sur leur vision de l'équipe à l'issue de la saison et sur l'indispensable adhésion des athlètes. A la différence des planifications proposées dans la littérature sur l'entraînement, leurs planifications apparaissaient plus souples ; elles pouvaient être remises en cause à tout instant. Les planifications étaient conçues pour le groupe, puis pour les individus. Les athlètes s'entraînaient une fois par jour. On ne connaissait ni leurs activités connexes à l'entraînement, ni l'incidence de ces activités sur l'entraînement, ni les particularités locales des conditions d'entraînement. Le temps apparaissait organisé par l'entraîneur, pour l'entraînement. Comme dans la littérature sur l'entraînement, le contexte était souvent «réduit» à : (a) une évaluation des aptitudes des athlètes, (b) aux objectifs poursuivis au plan sportif et à la spécificité du sport considéré, et (c) à leur motivation. Les aspects inhérents à l'histoire des agents (i.e. les athlètes et les entraîneurs), à leur vie au delà du sport, à leurs projets au delà des résultats sportifs semblaient occultés. Pourtant, ces aspects paraissent importants, car ils contribuent à déterminer la disponibilité et l'investissement des athlètes à l'égard de l'entraînement au quotidien. Un athlète qui s'entraîne tout en étant engagé dans une formation ou une activité professionnelle ne présente pas la même disponibilité et la même «fraîcheur » à l'égard de l'entraînement au regard d'un athlète dont l'activité essentielle est l'entraînement. Les propos d'un entraîneur cités en introduction permettaient d'illustrer ces idées.

Par ailleurs, les aspects liés à l'organisation de la structure dans laquelle sont réalisés les entraînements n'apparaissaient pas ou peu dans l'étude de Salmela (1996). En fait, cette activité de planification se focalisait le plus souvent sur la fixation de buts liés au sport considéré et de moyens pour les atteindre. Au delà de cette activité de planification de l'entraînement, l'observation des activités d'entraînement révèle une organisation des temps, qui s'appuie sur des éléments liés aux agents, aux institutions et aux connaissances sur le processus d'entraînement. Ces éléments ne semblent pas être considérés de façon isolée, mais plutôt de façon combinée et interactive. Il apparaît difficile d'occulter les éléments liés à la «vie extrasportive» des athlètes: les formations suivies, l'activité professionnelle, les situations familiales, personnelles... Ces éléments font partie de la vie des athlètes, ils peuvent avoir des incidences sur l'entraînement et sur la performance à certains moments de la vie (Mignon, 2007). L'organisation des temps dans les activités d'entraînement semble révéler des tensions, des résistances, qui pourraient être à l'origine de compromis entre les différents éléments considérés : la fréquence, l'enjeu des compétitions et leur ancrage dans le temps, les projets des athlètes et des institutions, la disponibilité des athlètes et des installations, les progrès des athlètes ou leurs régressions. Dans ces conditions, le temps n'apparaît pas unique et continu (Bergson, 1981), mais plutôt multiple et discontinu. Ce temps discontinu, ce «temps vécu » consiste en une pluralité de données qui n'ont ni le même rythme, ni le même enchaînement, ni la même puissance de contenus (Bachelard, 1936). Aux temps lisses et bien définis anticipés à travers une planification rationnelle, se distinguent des temps rugueux, chaotiques, mal définis, aux contours flous, présentant des relations d'imbrication, de superposition, de concurrence (Audigier, 2007). Ces temps rugueux sont perceptibles à travers l'observation des activités d'entraînement, ils reflètent des tensions, des imprévus, des concurrences entre les temps prévus pour les différentes activités. 


\subsection{Des micro-organisations temporelles locales au sein de l'entraînement}

Des études menées à partir d'approches sur l'action située (Suchman, 1987) et la cognition située (Kirschner \& Whitson, 1997) se sont centrées sur un temps plus court, celui de l'entraînement. Elles se sont focalisées sur certaines composantes temporelles de l'action des entraîneurs experts en tennis de table (Sève \& Durand, 1999) et en voile (Saury \& Durand, 1997). Elles ont permis d'identifier une dynamique temporelle dans les actions typiques réalisées par les entraîneurs. Cette dynamique n'était pas liée à une planification préalable, mais plutôt à l'émergence d'une organisation des actions fondée sur un couplage dynamique entre l'entraîneur et les éléments significatifs de la situation. Les actions s'ancraient dans des temporalités variées, liées aux différentes unités de travail considérées : l'action immédiate, les actions passées dans la séance, dans les séances précédentes, les actions à venir dans la séance ou au delà de celle-ci. Les résultats ont montré des temporalités entremêlées : des temporalités contraintes par les compétitions importantes (e.g., travail des points faibles ou des points forts), des temporalités liées à la progression de l'athlète, qui peuvent présenter des contradictions avec les premières. Ces études ont indiqué des phénomènes d'enchâssement des temporalités, à un niveau très local. Ces études se sont centrées sur des temps très courts. Il apparaît intéressant de se focaliser sur des temps plus longs, afin d'étudier l'organisation de ces temps dans un cadre contraint par les institutions dans lesquelles s'organisent les temps et les activités des agents.

\subsection{Des organisations temporelles dans une structure institutionnelle}

En France, l'entraînement des athlètes de haut niveau est majoritairement organisé au sein d'une structure d'état particulière : l'Institut National du Sport et de l'Education Physique (INSEP). Les deux tiers des médailles olympiques et $50 \%$ des athlètes olympiques participant aux Jeux Olympiques s'entraînent à l'INSEP (discours de Mme la Ministre de la Santé de la Jeunesse et des Sports du 7/07/2007). Le projet de l'institut consiste à mettre en place pour les athlètes, les conditions de la réussite sportive, scolaire et professionnelle (Ministère de la Santé de la Jeunesse et des sport, 2007 ; Bouchetal-Pellegri, Leseur \& Debois, 2007). Le temps est structuré par l'institut pour permettre aux athlètes de s'entraîner, mais aussi de se former, de se soigner, de s'alimenter, de se reposer. Ces temps sont organisés pour la journée, la semaine, l'année. Ces temps encadrent les activités des agents. La structuration des temps est discutée par les représentants des acteurs, puis utilisée comme cadre de référence pour l'ensemble des acteurs. En référence aux travaux de Dubar (2004) et d'Audigier (2007), les temps sont considérés comme étant subis au quotidien par les agents. Ils se distinguent des temps vécus, qui sont des temps singuliers, des temps de l'instantané. Cette pluralité des temps révèle une vision des temps propre à chacun d'entre nous. Les temps concernent à la fois le développement d'un être social dans la relation passé présent avenir et une échelle chronométrique déterminée par convention. Le temps apparaît découpé en portions. Chaque portion se réfère à un cadre temporel (Valax, 1986 ; Grossin, 1996). Les cadres temporels sont multiples, ils correspondent à un découpage conventionnel du temps : une olympiade, une saison, un cycle, une semaine, une journée. Ils sont structurés par l'organisation économique et sociale dans laquelle ils s'inscrivent. Ainsi, les cadres temporels de la semaine sont déterminés par la disponibilité des athlètes et des installations sportives à certains moments de la journée et de la semaine. La disponibilité des athlètes en faveur de l'entraînement est directement liée à leurs activités connexes, telles celles liées à la formation scolaire ou professionnelle, à l'exercice d'un métier, aux soins. Les cadres temporels apparaissent comme 
une voie d'entrée possible dans la construction des temps dans les activités d'entraînement. Mais comment se construisent ces temps dans les activités d'entraînement ?

\subsection{Deux conceptions distinctes du temps à l'origine de modes d'organisation particuliers}

Dupuy (2002) insiste sur la nécessité de retourner sur le passé pour comprendre sa vie et de se projeter dans l'avenir pour vivre cette vie. La vie est ancrée à la fois dans le passé et dans l'avenir. Il propose deux modes particuliers de construction du temps, en référence à deux conceptions distinctes du temps : le temps de l'histoire et le temps du projet. Dans la première conception, la prévision vise à simuler des phénomènes, à partir d'un modèle de déroulement causal. Le passé est considéré comme fixe et l'avenir est ouvert. Nos actions présentes ne peuvent avoir d'effet causal sur le passé et, par déduction, le passé ne dépend pas du présent. De plus, «nos actions présentes peuvent avoir un effet causal sur l'avenir et nous en inférons que l'avenir dépend contrefactuellement du présent » (p. 183). Le temps peut être représenté par un arbre dont les ramifications représentent l'avenir. Cette conception est utilisée par les stratèges, elle est à l'origine de l'élaboration d'arbres de la décision. Cette conception fondée sur un passé fixe et un avenir ouvert correspond à une conception «spontanée » du temps. Les agents se coordonnent à partir des actions passées et des engagements pris dans le passé.

Dans la seconde conception du temps dénommée le temps du projet, le futur est tenu pour fixe. Une anticipation particulière de l'avenir a des conséquences sur le passé qui l'anticipe et qui réagit en retour. Le temps représente une boucle dans laquelle le passé et l'avenir se déterminent réciproquement. L'avenir est envisagé tel qu'il est et non tel qu'il pourrait être. La prédiction n'est pas envisagée à partir d'un enchaînement causal des phénomènes, comme c'est le cas dans le temps de l'histoire. "C'est la prédiction consciente de son effet sur l'avenir, qui prédit l'avenir comme s'il était fixe et en même temps causé, au moins en partie par les effets de la prédiction » (p. 193). Ainsi, une anticipation de l'avenir peut concerner l'état de forme optimale des athlètes aux des Championnats du Monde. Les conséquences de cette anticipation renvoient aux moyens mis en ouvre pour atteindre cette anticipation: périodes d'entraînement, charge et volume d'entraînement, compétitions préparatoires... Le temps du projet contient en lui-même une idée de liberté : la liberté de prévoir n'importe quel avenir et d'en inférer les conclusions sur le passé qui l'anticipe et qui réagit à sa prédiction. Dans le temps du projet, avenir et passé sont co-déterminés. Les agents se coordonnent à partir d'une image de l'avenir, qui permet « d'assurer le bouclage entre une production causale de l'avenir et son anticipation autoréalisatrice » (p. 197).

\subsection{Les buts de l'étude}

L'étude poursuit trois buts. Le premier consiste à comprendre et à expliquer le processus de génération des organisations temporelles dans les activités d'entraînement ainsi que les éléments mobilisés dans ce processus. Le deuxième vise à analyser les interactions entre les éléments mobilisés. Le troisième consiste à révéler les organisations temporelles issues de ce processus ainsi que leurs relations. 


\section{Méthode}

\subsection{Les participants.}

Dix hommes, entraîneurs nationaux de l'INSEP ont participé volontairement à l'étude. Ils entraînaient depuis au minimum dix ans des athlètes de haut niveau. Ils étaient âgés entre 36 et 59 ans (âge moyen $=47$ ans, écart type $=7$ ans et 10 mois). Ils étaient amenés à s'inscrire dans la structuration des temps de l'institut et des fédérations sportives, pour contribuer à organiser les temps dans les activités d'entraînement. Ils évoluaient dans des sports variés : cyclisme, gymnastique, natation, basket-ball, badminton, escrime, lutte, judo, athlétisme (deux entraîneurs concernés).

\subsection{Le recueil des données}

Dix entretiens d'1h30 environ ont été menés, à partir d'un guide. Les questions portaient sur l'organisation du temps dans les activités d'entraînement, au cours de la saison, dans un institut dans lequel des plages horaires sont destinées aux différentes activités quotidiennes. Les questions partaient d'un commentaire du calendrier, de l'agenda ou de programmations réalisées sur feuilles excel. Ces documents constituaient des traces de l'activité utiles à l'analyse (voir figure 1). Ils étaient utilisés comme déclencheur permettant à l'entraîneur de se confronter avec sa pratique et de la verbaliser (Jeannot, 2000). L'entraîneur était amené à commenter ces traces et à décrire l'activité concomitante d'organisation des temps au cours de la saison. L'évocation d'un temps particulier ou d'une période appelait des commentaires particuliers sur leurs significations, leurs origines et leurs mises en œuvre. Les relances portaient sur le calendrier sportif et les événements prioritaires (e.g., "Quels événements prends-tu en compte de façon prioritaire?»), sur les moments importants de la saison (e.g., «Quels sont les moments particulièrement importants, sur le découpage du temps au cours de la saison (e.g., « Comment découpes-tu la saison? la période ?), sur les moments du travail individuel ou collectif avec les athlètes (e.g., « Ce planning concerne-t-il tous les athlètes du groupe ?») 
Figure 1 : Planning

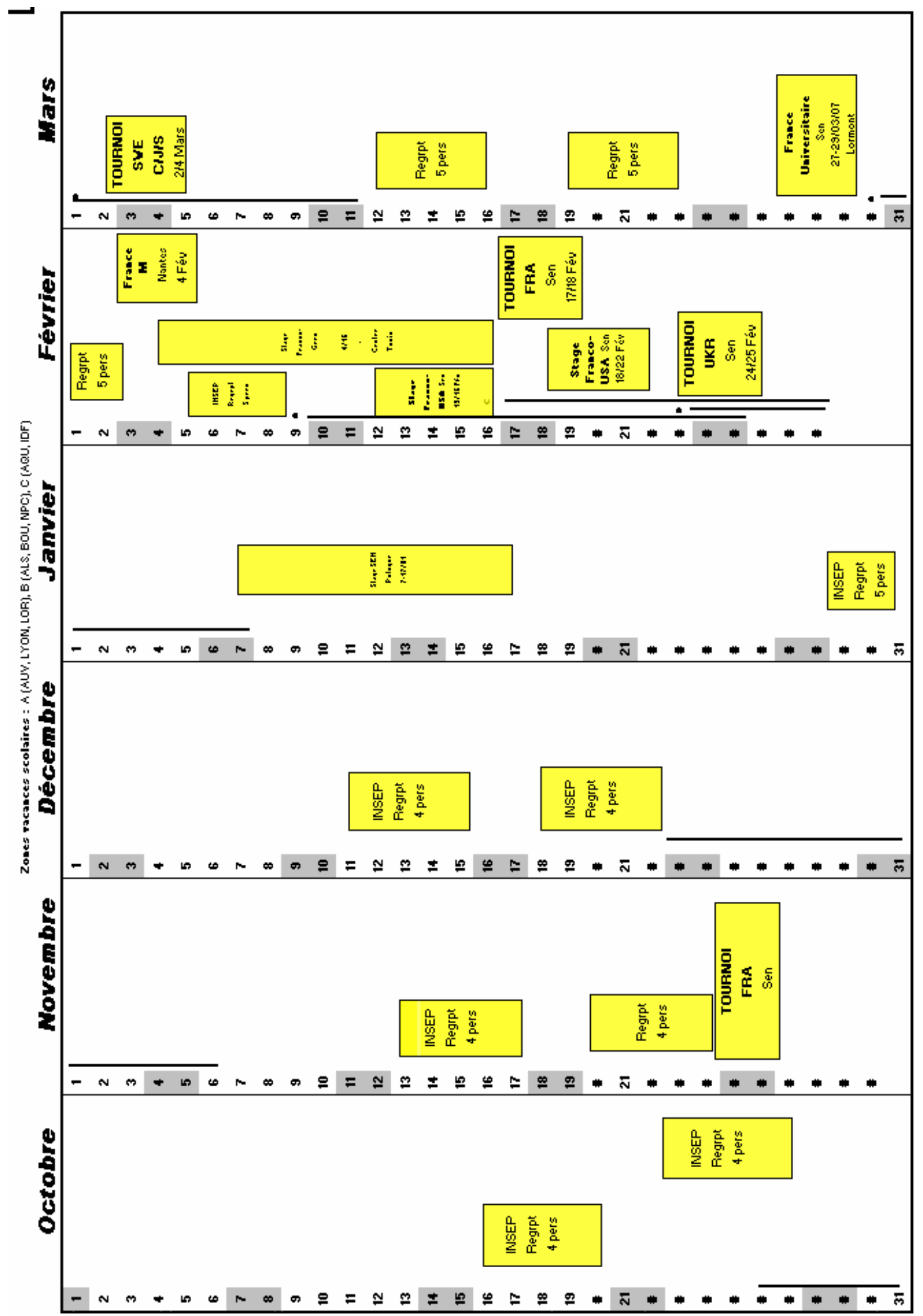




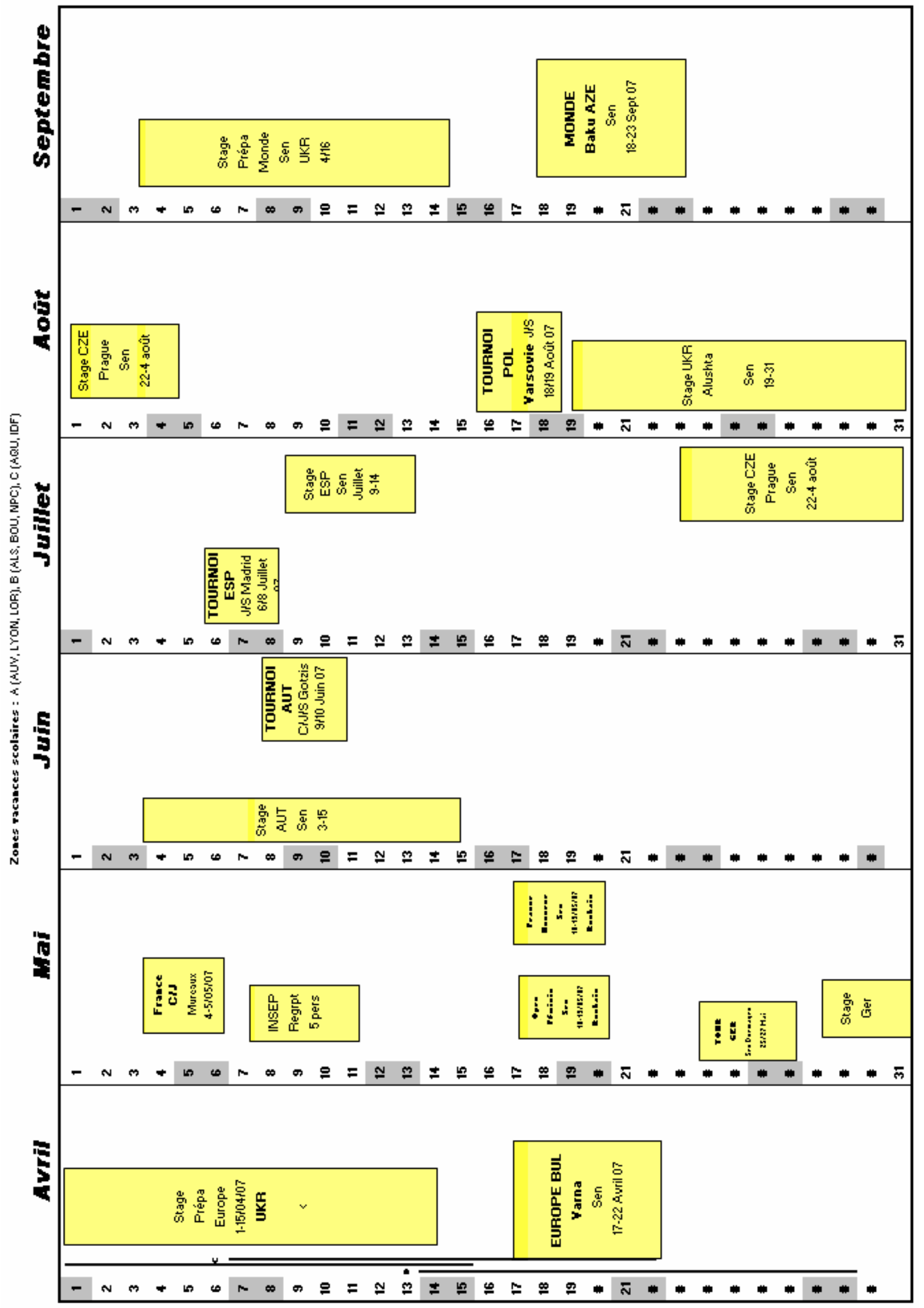




\subsection{Le traitement des données.}

Les entretiens ont été retranscrits verbatim. Les verbalisations ont fait l'objet d'analyses inductive et déductive à partir de la théorie ancrée (Corbin \& Strauss, 1990). Ces analyses sont progressives. Les retranscriptions sont divisées en unités de sens, par deux chercheurs indépendants, formés aux méthodes qualitatives. Ces unités sont ensuite comparées et discutées entre les chercheurs pour obtenir un consensus. Elles sont alors comparées et regroupées en catégories de plus en plus larges, puis définies en fonction de leur contenu. La définition et le contenu des catégories sont ensuite discutés afin d'obtenir un consensus entre les chercheurs. Enfin, les catégories sont analysées afin d'identifier leur relations et leurs différentiations, dans un souci de cohérence. Chaque entretien est analysé progressivement. Les entretiens sont étudiés de façon successive. Dans ces conditions, on peut prévoir que les derniers entretiens ne permettent pas d'identifier de nouvelles catégories. Ceci permettra d'indiquer que le «seuil de saturation qualitative» des données pour ces participants aura été atteint. Les résultats seront ensuite soumis aux entraîneurs, pour obtenir leur avis. 


\section{Résultats}

Trois types de résultats ont été obtenus. Ils renvoient à trois catégories issues des analyses inductive et déductive des retranscriptions. La première a porté sur le processus de génération des organisations temporelles dans les activités d'entraînement. La deuxième a mis en évidence une activité de compromis liée à des tensions et des résistances entre les éléments mobilisés dans l'organisation des temps. La troisième a consisté en l'identification d'organisations temporelles typiques générées en fonction des activités sportives considérées.

\subsection{Le processus de génération des organisations temporelles}

Le premier type de résultat a montré que les organisations temporelles sont générées par un processus circulaire, qui consistait en une anticipation de l'avenir et en un retour vers le passé. Ce processus s'appuyait sur trois paramètres en interaction: les compétitions, les projets et l'entraînement.

\subsubsection{Un processus circulaire}

Ce processus s'appuyait sur une projection vers l'avenir visant à anticiper le niveau de performance optimal des athlètes lors des compétitions. Le retour vers le passé s'accompagnait de la mobilisation de paramètres afin de réaliser cette anticipation de l'avenir. Des verbatim ont permis d'illustrer ce processus circulaire :

«La première chose que j'organise, c'est la saison. Je prépare la saison, les moments importants, les différents cycles. En fait, il faut que je mette tout en relation [...] Les premiers objectifs c'est les compétitions internationales de référence: championnats d'Europe, Jeux Olympiques, championnats du Monde. Le deuxième objectif, c'est la façon dont on s'y qualifie, donc c'est les Championnats de France. Et après, tout le reste, ça s'articule par rapport à ces deux compétitions là. [...] En fait, il y a une compétition par an. Des fois, y'en a deux, quand y'a les Jeux et les Europe. Y'a une compétition l'été [...] Donc après, je construis une saison, je fais un calendrier où je fais toute la saison, les week-end de repos... Avec cette démarche, je sais que l'athlète sera en forme par rapport à ses qualités physiques lors de la compétition. » natation

"C'est à nous de déterminer un calendrier par rapport au calendrier international, de faire ça en cascade, de manière à ce qu'il y ait une programmation qui soit sportivement cohérente [...] arriver à avoir une préparation sportive qui les amène dans les meilleures conditions possibles aux grands événements et ça plusieurs fois dans l'année. » escrime

Dans ces verbatim, l'anticipation portait sur le niveau de préparation sportive et sur l'état de forme lors des compétitions majeures. Le retour vers le passé s'accompagnait d'une prise en compte des compétitions situées avant les compétitions majeures, et qui s'articulaient à ces dernières (e.g., qualification). Une projection dans l'avenir était ensuite réalisée afin de construire l'ensemble de la saison. Ce processus circulaire s'appuyait sur trois paramètres en interaction. 


\subsubsection{Trois paramètres en interaction}

Ces paramètres ont été présentés de façon isolée afin de mieux les identifier. Ils étaient mobilisés de façon conjointe au sein du processus et interagissaient les uns avec les autres. Ils consistaient en: (a) les compétitions, (b) les projets et (c) l'entraînement. Le paramètre « compétitions » renvoyait aux événements sportifs. Le paramètre «projets » concernait les agents et les institutions; il incluait le sport et les activités au delà du sport; il consistait en des objectifs et en des moyens pour les atteindre. Le paramètre «entraînement » renvoyait à l'ensemble des connaissances sur l'entraînement utilisées pour organiser le travail et le repos, au regard des objectifs sportifs poursuivis dans la durée. Chaque paramètre révélait des composantes particulières, qui pouvaient présenter des tensions, des résistances avec d'autres composantes du même paramètre ou des composantes d'autres paramètres. Treize composantes ont été mises en évidence à travers les trois paramètres. Elles ont été présentées avec leur paramètre correspondant.

\subsubsection{Les compétitions}

Ce paramètre concernait les éléments liés à la compétition. La compétition apparaissait à la fois comme un événement au cours de la saison et un objectif pour les athlètes et les entraîneurs. Les athlètes étaient amenés à préparer cet objectif en vue de réaliser des performances. Ce paramètre présentait trois composantes : (a) les dates des compétitions, (b) le type de compétition, qui est associé à des objectifs et à des enjeux particuliers et (c) le format de la compétition, c'est-à-dire le programme des épreuves.

Les dates des compétitions

Elles concernaient les compétitions prévues par les fédérations internationales et nationales. Des verbatim ont permis d'illustrer cette composante :

«les championnats d'Europe c'est en avril, les championnats du monde, c'est en septembre octobre. » lutte

«Il y a une épreuve de coupe du monde par mois entre octobre et janvier, le championnat de France en août et les championnats du monde en octobre» cyclisme

\section{Le type de compétition}

Il était associé à des objectifs et à des enjeux particuliers. Cette composante renvoyait à l'importance de la compétition au regard de la saison, ses enjeux pour les athlètes au plan des compétitions suivantes et l'objectif qui lui était assigné pour les athlètes. L'objectif pouvait être une qualification ou une sélection pour une compétition ultérieure, à une volonté de se montrer face aux concurrents et éventuellement aux juges. Des verbatim ont permis d'illustrer cette composante :

"y'a des compétitions principales et des compétitions secondaires. Les compétitions principales sont des repères, il faut qu'ils se montrent par rapport à l'adversité, ils vont éventuellement chopper une sélection ou se qualifier pour le tour d'après. La principale étant le championnat de France, pour se qualifier aux différents championnats qu'il y a après. Les compétitions secondaires sont des entraînements spécifiques, ce sont des compétitions dites de travail, elles doivent être vécues comme des entraînements. Les compétitions principales sont de véritables tests. » athlétisme 
«Le tournoi de Manheim [...]Pour moi, c'est un virtuel championnat du monde. Ca m'intéresse parce que ça va me renseigner par rapport aux championnats d'Europe [...] on peut voir quels joueurs peuvent jouer. » basket-ball

«y'a un paramètre de la performance gymnique qui s'appelle la notoriété. C'est d'avoir été reconnu dans plusieurs tournois pour avoir une chance de faire un résultat. Les gym se font un nom [...] ça leur donne une certaine valeur » gymnastique

L'objectif poursuivi à travers la compétition pouvait être lié à l'organisation même de la compétition (i.e. athlétisme) ou à l'organisation mise en place pour le groupe d'entraînement (i.e. basket-ball). Il pouvait également être lié au mode d'évaluation du sport considéré, comme en gymnastique.

\section{Le format de la compétition}

Cette composante renvoie au programme de la compétition. Elle n'est apparue qu'en gymnastique. Des verbatim ont permis de l'identifier :

"les compétitions sont compétitions individuelles ou par équipe. Ce n'est pas le même travail. Si c'est un tournoi par spécialité, c'est encore un autre travail. S'ils travaillent sur les six agrès, l'objectif n'est pas le même que s'ils travaillent sur deux agrès » gym

Le format de compétition amène à organiser le travail de façon différenciée dans le temps.

\subsubsection{Les projets}

Le paramètre lié aux projets consistait en deux composantes : l'une était liée aux projets et aux attentes des institutions à l'égard des agents et l'autre était inhérente aux projets des agents. Les institutions étaient les fédérations sportives et l'INSEP. Les agents étaient les athlètes et les entraîneurs. Les projets apparaissaient singuliers, même s'ils présentaient des similitudes.

\section{Les projets des institutions}

Ils concernaient trois domaines : (a) la mise en œuvre de conditions optimales pour organiser la vie des athlètes, (b) l'obtention de résultats sportifs et (c) la gestion d'un budget pour le groupe d'entraînement.

La mise en ouvre de conditions optimales pour organiser la vie des athlètes

Cet élément s'inscrivait dans une volonté de concilier le projet sportif de l'athlète et le doublement de ce projet à travers une formation scolaire, universitaire ou professionnelle, une activité professionnelle ou des activités familiales (pour les mères de famille). Des verbatim ont permis d'illustrer cet élément :

" avoir une préparation sportive et pouvoir en même temps suivre les cours, aller au boulot et se reposer $»$ escrime

"Y'a deux objectifs : préparer la saison pour les championnats d'Europe et préparer les gamins à devenir des professionnels. » basket-ball

Dans le cas du basket-ball, les objectifs étaient centrés sur le sport : le premier renvoyait aux résultats sportifs du groupe à l'issue de la saison et le second concernait la formation 
professionnelle de chacun des joueurs, finalisée, de façon implicite dans les verbatim, par le recrutement par un club professionnel.

L'obtention de résultats sportifs

Cet élément s'articulait avec le paramètre compétition. Il concernait les attentes de résultats par la fédération sportive. Des verbatim permettaient de l'illustrer :

«on va nous demander de maintenir nos cinq médailles aux championnats d'Europe et d'esquisser un truc pour avoir des résultats chez les juniors et les cadettes pour avoir plus de moyens », lutte

«Aux championnats d'Europe, il fallait avoir une médaille [...] y'a des objectifs comme ça qui sont annoncés ». badminton

L'obtention de résultats apparaissait liée à l'élément concernant la gestion du budget.

La gestion d'un budget pour le groupe d'entraînement

Elle renvoyait aux contraintes financières imposées par la fédération sportive pour organiser l'entraînement dans le pôle et hors du pôle, les compétitions et les stages. Le budget évoluait en fonction des résultats obtenus la saison précédente. Des verbatim permettaient de l'illustrer :

«Tout en découle, à un moment, le budget que le Ministère donnait à la fédération, c'était grâce au sprint, parce qu'il a ramené 4 à 5 médailles aux Jeux. Quand vous passez à la formation d'objectifs et que vous avez ça sur la table, on refuse un peu moins à des gens comme nous qu'à des fédérations qui se sont loupées. [...]Le DTN demande à chaque entraîneur de faire son planning de compétitions et de stages. Et là on budgétise. Une fois qu'il a ça, il fait un petit calcul et puis il voit avec le Ministère pour la convention en disant " oh la la la, là c'est trop », alors hop on retourne et là il appelle chaque entraîneur en disant " ben là, il faut que tu supprimes quelque chose, donc il y a des coupes sombres, s'il n'a pas obtenu le budget qu'il voulait. » cyclisme

\section{Les projets des agents}

Ils renvoyaient au projet sportif et aux projets liés à la formation, aux activités professionnelles ou familiales. Ils concernaient trois domaines: (a) les résultats sportifs attendus par les agents, (b) la disponibilité des athlètes et (c) les gains financiers envisagés par les athlètes.

Les résultats sportifs attendus par les agents

Ces résultats étaient attendus par les entraîneurs et les athlètes. Ils concernaient les résultats proprement dits et l'anticipation de résultats au regard du niveau d'adversité. Des verbatim ont permis d'illustrer les résultats attendus :

«La joueuse est sur un objectif de performance aux Jeux, d'être si possible dans les quatre premières têtes de série [...]Il faut avoir dix gros résultats [...] La joueuse n'est que sur des compétitions tops, les autres compétitions ça ne l'intéresse pas parce c'est inférieur à sa moyenne. » badminton 
D'autres verbatim ont permis d'illustrer l'anticipation des résultats au regard du niveau d'adversité :

«Ce qui est important, c'est d'avoir des informations sur la participation des chinoises, parce que quand elles ne sont pas là, elle a plus de chances de gagner . » badminton

\section{La disponibilité des athlètes}

Cet élément renvoyait directement aux activités de chaque athlète incluant ses activités liées à l'entraînement et à celles non directement liées à l'entraînement. Ces dernières influaient sur l'organisation du temps lié aux activités d'entraînement. Des verbatim ont permis de l'illustrer :

«J'ai des athlètes mariées, qui ont des enfants » Lutte

«J'ai des athlètes qui travaillent d'autres qui sont en fac à l'extérieur » escrime

«J'organise le temps en fonction de leurs emplois du temps au quotidien » athlétisme

Pour de nombreux athlètes, sport de haut niveau et vie s'organisaient au sein de l'INSEP. Pour d'autres, le sport de haut niveau s'organisait avec une vie familiale, une activité professionnelle ou une formation réalisée à l'extérieur de l'INSEP, qui amenait à une organisation différenciée des temps et une individualisation des temps de l'entraînement.

\section{Les gains financiers envisagés par les athlètes}

L'inscription à certaines compétitions pour certains athlètes et / ou les résultats obtenus lors de ces compétitions permettent aux athlètes d'obtenir des gains financiers. Ces gains potentiels ou réels présentent une motivation importante pour les sportifs qui peuvent être amenés à privilégier certaines compétitions au regard d'autres, ou à augmenter le nombre de compétitions, ce qui peut influer sur les temps de préparation et de repos et sur le budget du groupe. Des verbatim ont permis d'illustrer ces gains financiers :

«elle gagne pas mal d'argent dans les tournois de badminton, contrairement aux autres joueurs. Comme elle gagne pas mal d'argent, c'est aussi une motivation importante pour elle. Gérer une athlète comme ça, c'est un peu différent car elle demande à participer à ces compétitions. Quand elle en rate, ça l'embête. » badminton

"Les tout meilleurs ont des teams internationaux auxquels ils doivent répondre pour être dans les classifications mondiales et être invités sur la finale mondiale en athlétisme, qui est intéressante financièrement; mais tous ne sont pas dans ce cas là. Il y a le côté national qui est intéressant par l'enjeu des rencontres et par cet aspect financier. » athlétisme

Cette possibilité de gains orientait le choix des athlètes vers certaines compétitions ou amenait les athlètes à vouloir augmenter le nombre de participations aux compétitions. Cet élément contribuait à un choix des compétitions pour l'individu. Cet élément concernait essentiellement l'athlétisme, le badminton et dans une moindre mesure le cyclisme.

\subsubsection{L' entraînement}

Il concernait les différents aspects de l'entraînement envisagés en relation avec les compétitions. Il renvoyait à des connaissances liées à la méthodologie de l'entraînement et à des connaissances liées à l'expérience. 
Il présentait quatre composantes : (a) le temps requis pour préparer les échéances et pour se reposer, (b) l'augmentation progressive de la charge et du volume de travail; (c) la détermination de temps pour un travail ou un effort particulier, (d) l'espace et la sécurité.

Le temps requis pour préparer les échéances et pour se reposer.

Cette composante renvoyait au temps nécessaire aux athlètes pour atteindre un état de forme optimal, pour apprendre de nouvelles techniques et tactiques en vue de réaliser des performances maximales et pour se reposer. Des verbatim ont permis d'illustrer cette composante :

«J'essaie de choisir des épreuves qu'on puisse préparer correctement, à savoir, il me faut au moins quatre semaines avant une épreuve importante que je vais considérer comme sélective. » escrime

«Pour faire une compétition de ce niveau là, il faut être prêt trois semaines avant [...] on fait beaucoup d'impasses car un des objectifs de notre métier c'est quand même de préserver l'intégrité physique de nos athlètes. » gymnastique

La durée de préparation ou de «re-préparation » variait selon les sports. Aux temps de préparation et de compétitions suivaient des temps de repos. Des verbatim permettaient de les illustrer :

«On met en place des périodes de travail. Y'avait les championnats du monde, puis du repos pour l'équipe de France. Ensuite, y'a eu les championnats d'Europe, on a mis un peu de liberté, donc on est parti, on a fait une reprise d'entraînement assez tranquille parce que de toute façon ils étaient entraînés, on n'a pas fait une préparation spécifique pour le championnat d'Europe à part les préparations tactiques parce qu'on peut jouer que là-dessus finalement. » judo

$\underline{\text { L'augmentation progressive de la charge et du volume d'entraînement }}$

Cette composante était identifiée à travers les commentaires des contenus d'entraînement. Elle renvoyait à une progressivité du travail avec les athlètes. Des verbatim ont permis d'illustrer cette composante :

«La saison est découpée en trois cycles [...] le cycle est simple : on se remet en condition, on fait de la force, on fait de la force endurance, après, on a tant de volume par semaine et après j'ai différents types de semaines ». natation

"On a le travail d'endurance spécifique. En septembre, on a le travail de base, de technique, dès le mois d'octobre, on va rentrer dans une période de mouvements complets, qui est aussi un travail d'éléments, qui est progressif, on rentre pas tout de suite dans la compétition. On fait un travail d'éléments, on travaille la moitié des mouvements, puis les mouvements complets. Ensuite on va essayer de maintenir ce travail pendant trois semaines. Au 15 octobre déjà, on est dans un travail de mouvement complet, ensuite on va essayer de maintenir ce travail pendant trois semaines, jusqu'aux championnats du monde. Voilà en gros comment se passe une préparation. Cette préparation à partir du mois d'août est beaucoup plus rapide que celle du retour de décembre, parce que l'échéance est beaucoup plus courte. Et comme les athlètes n'ont pas arrêté, ils ont l'acquis du premier semestre [...] »gymnastique 
«On a grosso modo 7, 8 semaines de la Toussaint à Noël, on va travailler pas mal le physique, j’introduis l'escrime disons la troisième semaine, de manière à reprendre doucement le travail individuel en fonction des gens, parce qu'il y a des gens qui n'en ont pas besoin autant que d'autres, notamment les meilleurs à qui je donne la leçon depuis quelques années, le travail pour eux est beaucoup plus un travail de reprise, après les championnats du monde [...] A partir de janvier, comme je vous l'ai dit, on est à trois semaines de l'épreuve, c'est du spécifique et là il faut éviter la blessure, il faut redonner en l'espace de trois semaines et c'est ça qui est intéressant, c'est qu'en trois semaines on arrive à retrouver notre optimum spécifique, aussi bien physique que technique... » escrime

L'augmentation de la charge et du volume d'entraînement étaient fonction du sport considéré, de la période de l'année et des athlètes. Elle dépendait du volume et de la charge précédemment choisie, du temps de repos entre deux compétitions, de l'état de forme des athlètes, de la durée séparant le présent de la prochaine compétition importante. Le travail est apparu cyclique. Chaque cycle était borné par une compétition majeure. Les verbalisations suggéraient une succession de phases liées à une préparation initiale, une préparation intermédiaire, une préparation terminale, à la ou aux compétitions et au repos. Les phases de préparation révélaient une progressivité dans le travail et le maintien d'un niveau de travail. Le repos était envisagé à la fin du cycle ou au cours du cycle au regard des compétitions successives. Dans le premier cas, le repos était souvent total, dans le second il était inclus dans les phases d'entraînement (e.g. «on récupère »).

\section{La détermination de temps pour un travail ou un effort particulier}

Cette composante indique que des temps particuliers sont consacrés à des apprentissages spécifiques, à une amélioration des points forts et à une réduction des points faibles, et à la réalisation d'un type d'effort particulier. Des verbatim ont permis de l'illustrer :

"pour ceux qui ont des objectifs précis, l'entraînement est à base de vélocité de force vitesse », cyclisme

«Les temps d'apprentissage c'est tout le temps, hormis les périodes de compétition, parce que là ils sont concentrés sur la perf. C'est une à trois semaines avant une compétition » judo

\section{L'espace et la sécurité des athlètes}

Cette composante concernait l'espace disponible au regard du nombre d'athlètes et des possibilités de maintenir des conditions de sécurité satisfaisantes pour les athlètes. Cette composante présentait des effets sur les moments et les durées d'entraînement pour chacun des athlètes et éventuellement sur le lieu précis de travail de chacun d'eux. Des verbatim ont permis d'illustrer cette composante :

«la première chose qu'on prend en compte c'est l'espace. On a un gymnase où on est 24, on a deux paniers centraux [...] on fait généralement deux groupes de niveau: un groupe qui fonctionne sur le terrain central et un groupe qui fonctionne à l'extérieur. Ensuite, on change. » basket-ball

«J'organise le temps en fonction des questions de sécurité, pour qu'ils ne soient pas plus de deux ou trois à l'entraînement » athlétisme

Elle est apparue le plus souvent en basket-ball, en athlétisme et en cyclisme et occasionnellement en judo et en natation. 
Le paramètre lié à la compétition était le premier mobilisé dans le processus. L'organisation des temps s'ancrait sur les dates fixées par les calendriers fédéraux. Très vite, les autres paramètres étaient mobilisés ainsi que leurs composantes. Si les trois paramètres apparaissaient dans tous les entretiens, certaines composantes n'étaient révélées que par certains entraîneurs. Ainsi, la composante liée au programme des compétitions n'était rapportée que par l'entraîneur de gymnastique. La composante liée à l'espace et à la sécurité était rapportée par les entraîneurs d'athlétisme, de basket-ball, de cyclisme et occasionnellement de judo et de natation. La composante inhérente aux gains financiers n'était rapportée que par les entraîneurs de badminton, d'athlétisme et de cyclisme. Les résultats ont montré des singularités dans la mobilisation des paramètres et de leurs composantes en fonction des sports et des agents (mères de famille, athlètes salariés, athlètes percevant des gains financiers grâce à leurs résultats sportifs).

Le premier type de résultats a montré que les organisations temporelles étaient générées à travers la mobilisation de trois paramètres en interaction. Les composantes de ces paramètres et les éléments de ces composantes ont contribué à identifier le cadre dans lequel les organisations étaient générées.

\subsection{Les organisations temporelles : des inventions locales issues de compromis}

Le deuxième type de résultats a montré que la mobilisation du processus de génération des organisations temporelles engendrait des tensions, des concurrences, des résistances entre les composantes des paramètres. L'entraîneur était amené à réaliser des compromis pour réduire ces tensions, ces concurrences et résistances. Six compromis ont été identifiés dans l'analyse: (a) sauvegarder du temps pour maintenir une préparation de qualité tout en répondant aux attentes des athlètes, (b) privilégier les compétitions sélectives tout en maintenant une durée de préparation optimale entre deux compétitions, (c) favoriser le retour dans les familles tout en préservant du temps pour préparer les compétitions, (d) individualiser l'entraînement tout en organisant le travail pour le groupe, (e) optimiser les temps d'entraînement tout en respectant les temps de l'institution (Insep), et (f) libérer des temps pour se reposer tout en occupant ces temps pour ne pas s'ennuyer.

3.2.1. Sauvegarder du temps pour maintenir une préparation de qualité tout en répondant aux attentes des athlètes

Le premier compromis résultait de la réduction de tensions et de concurrence entre l'objectif de préparation d'un événement et la volonté de partager du temps avec sa famille ou de consacrer du temps à son activité professionnelle ou de formation scolaire ou universitaire. Il était lié à une concurrence entre le projet sportif de l'athlète et les activités connexes à ce projet. Des verbatim illustraient cette concurrence et les compromis réalisés avec des athlètes, mères de famille :

"J'ai des athlètes mariées, qui ont des enfants, donc ce n'est pas du tout la même approche que si elle est célibataire jeune et qu'elle est au pôle. Je suis conscient que ça leur en demande plus, je dois donc aménager. Après, y'a une négociation qui se passe, on va dire comme un accord mais qui doit être par rapport à l'objectif. C'est assez difficile, je dirais qu'on arrive à des situations où les athlètes voudraient pratiquement faire quinze jours de préparation et puis aller directement aux Championnats d'Europe [...]Je crois qu'il faut être 
vigilant, y'a des négociations qui sont possibles, des discussions possibles, mais après il faut s'arrêter sur un cadrage minimum du travail à faire. On a trois athlètes qui sont dans ce cas et qui vivent en province, qui passent par l'INSEP pour des stages de regroupement [...] Pour participer aux Championnats d'Europe, il faut au minimum deux tournois, plus on va dire trois semaines de préparation, un mois de préparation convenable, en sachant bien sûr qu'il y a une période antérieure, elle va peut-être se préparer de son côté. »

En lutte, le projet lié à l'obtention de médailles aux championnats d'Europe entrait en concurrence avec le projet des athlètes mères de famille. L'éloignement du lieu d'entraînement au pôle France au regard du lieu de vie de leur famille amenait les athlètes à s'absenter de leur domicile et à distinguer dans le temps, les activités sportives en vue de la préparation aux compétitions majeures et les activités familiales. Certaines athlètes souhaitaient réduire le temps de préparation aux événements majeurs. L'entraîneur était alors amené à négocier avec ces athlètes. Ils trouvaient des compromis, pour sauvegarder du temps afin de maintenir une préparation de qualité, tout en répondant partiellement à leurs attentes respectives (libérer du temps / avoir suffisamment de temps pour se préparer correctement). Ces compromis concernaient essentiellement les athlètes dont les activités en dehors de l'entraînement étaient réalisées à l'extérieur de l'INSEP : formations universitaires différentes de celles proposées par l'INSEP, activités professionnelles, familiales. Ces athlètes s'entraînaient en lutte, en escrime et en athlétisme.

3.2.2. Privilégier les compétitions sélectives tout en maintenant une durée de préparation optimale entre deux compétitions

Le compromis consistant à privilégier les compétitions sélectives tout en maintenant une durée de préparation optimale entre deux compétitions visait à réduire les tensions entre d'une part, les dates des compétitions proposées par le calendrier international et le type de compétition et d'autre part, le temps nécessaire pour préparer efficacement ces échéances et pour se reposer. Ce compromis pouvait être illustré à partir de verbatim :

«De janvier à juin, il y a la grosse période de sélection, où il y a le plus d'épreuves de coupe du monde. Ces épreuves de coupe du monde, à moi d'en choisir quelques une que je vais prendre comme compétition de sélection pour les Championnats du Monde ou pour les Europe. [...]J'essaie de choisir des épreuves qu'on puisse préparer correctement, à savoir, il me faut au moins quatre semaines avant une épreuve importante que je vais considérer comme sélective.». escrime

L'entraîneur était alors amené à choisir suffisamment de compétitions rapprochées dans le temps, pour permettre aux athlètes d'être sélectionnés dans des compétitions majeures, tout en leur donnant quatre semaines pour se reposer après une échéance et pour préparer la suivante. Parfois, ce compromis ne pouvait être trouvé et l'entraîneur était amené à reformuler le problème en insistant sur la finalité de la préparation : les résultats aux compétitions. Cette reformulation l'amenait à privilégier les compétitions sélectives :

«il y a souvent des couacs: fin janvier début février j’avais deux épreuves très très importantes, j'avais ce qu'on appelle les grands prix, on est sûr qu'il y a beaucoup de monde, je les ai pris comme épreuve sélectives, c'était les seules, donc j'avais vraiment pas le choix. » escrime 
Les compromis ne résultaient pas seulement d'un «arrangement » entre des paramètres ou des composantes des paramètres en concurrence. Ils étaient issus de la priorité donnée à une de ces composantes ou plus spécifiquement à un objectif particulier tel celui d'obtenir des résultats en vue de se qualifier pour les Championnats du Monde ou d'Europe. Cet objectif paraissait être le fil directeur de l'organisation des temps au cours d'une période. Ainsi, les compromis étaient trouvés au regard de l'objectif de résultats.

3.2.3. Favoriser le retour dans les familles tout en préservant du temps pour préparer les compétitions

Un autre compromis consistait à favoriser le retour dans les familles tout en préservant du temps pour préparer les compétitions. Le retour dans les familles prenait du temps et ce temps était souvent pris sur le temps d'entraînement. Ce temps nécessaire au retour variait en fonction de l'éloignement des familles au regard du lieu d'entraînement. Parfois, les tensions, les concurrences entre ces temps faisaient l'objet de compromis pour certains athlètes. Des verbatim permettaient d'illustrer ce compromis :

«ça pose quand même le problème des antillais qui ne peuvent pas rentrer sur des périodes courtes. La plupart ont de la famille en métropole ». basket-ball

"j'essaie à chaque fois de leur donner du temps pour rentrer chez eux parce que tu ne mets pas à égalité celui qui habite la région parisienne avec des garçons qui rentrent une seule fois par an chez eux dans les DOM». escrime

Les entraîneurs tentaient de réaliser des compromis pour favoriser le retour, tant que ce retour ne risquait pas de nuire au temps de préparation aux différents événements. La réalisation de compromis n'était possible que si elle s'inscrivait dans la poursuite des objectifs sportifs.

\subsubsection{Individualiser l'entraînement tout en organisant le travail pour le groupe}

Un autre compromis consistait à individualiser l'entraînement tout en organisant le travail pour le groupe. Dans ce cas, la composante liée aux échéances compétitives présentait des tensions avec les composantes liées au type de compétition, aux projets des agents, aux temps requis pour déterminer les échéances, à la détermination de temps pour un travail ou un effort particulier, à l'augmentation progressive de la charge. Des verbatim révélaient que la multiplication des compétitions pour les athlètes amenait l'entraîneur à réaliser des entraînements individuels dans le cadre d'un collectif :

«... ce sont des cycles qui sont réservés à des coureurs qui n’ont pas d'objectif. Celui qui va faire la coupe du monde ne va pas faire ce cycle là. Donc ils vont avoir des cycles différents selon les objectifs qu'ils vont faire. Avant c'était bien clair, on avait le championnat du monde au mois d'août et les coupes du monde en avril, mai, juin, on avait un travail foncier de septembre à février et puis après on avait un cycle de pré-compétition et un cycle de compétition, on avait trois grands cycles alors que maintenant... C'est presque un entraînement individuel avec des cycles en fonction des objectifs.». cyclisme

L'entraîneur était amené à trouver un optimum dans l'organisation du temps, afin de préserver les temps de préparation pour chacun et de maintenir une qualité dans chacune des préparations. D'une structuration du temps de la saison pour le groupe d'entraînement, on est passé à une structuration du temps pour chacun des athlètes. 
3.2.5. Optimiser les temps d'entraînement tout en respectant les temps de l'institution (INSEP)

Un autre compromis consistait à optimiser les temps d'entraînement tout en respectant les temps de l'institution (INSEP). Ce compromis visait à réduire les concurrences entre les temps liés à l'organisation sportive et ceux liés à l'organisation institutionnelle (INSEP). Si l'institution prévoyait des temps pour chaque activité, ces temps se bousculaient. Ainsi, une concurrence entre les temps de l'entraînement et les temps hors de l'entraînement permettaient d'illustrer ce compromis :

«c'est simple, quand on est en stage, il y a le judo, l'entraînement, les objectifs liés à ça et la vie quotidienne qui passe après presque, c'est d'abord centré sur l'entraînement, c'est pour ça que c'est payant. Quand on est à l'INSEP, c'est d'abord leur vie, leur quotidien et ensuite dans leur quotidien, il y a l'entraînement. » judo

«L'organisation de ma semaine va dépendre des contraintes de formation. Je préférerais qu'ils nagent à 8 heures, mais ici c'est pas possible.» natation

"Le vendredi matin, ils vont en cours à l'extérieur donc on n'a pas d'entraînement. On a mis un entraînement plus long l'après-midi. » basket-ball

Les priorités données aux différents temps variaient. Elles ont permis de distinguer des temps exclusivement centrés sur l'entrainement, de temps à focalisations variables, induits par des contraintes institutionnelles fortes. Les propos révélaient une concurrence importante entre des temps de l'institution liés à la formation scolaire, universitaire et professionnelle des athlètes, et les temps de l'entraînement finalisés par les résultats sportifs au plan international.

3.2.6. Libérer des temps pour se reposer tout en occupant ces temps pour ne pas s'ennuyer

Les stages réalisés à l'extérieur de l'INSEP entraînaient une «libération » des temps de formation et d'activité salariée. Cette «libération» de ces temps induisait parfois la génération d' autres temps, comme des temps d'ennui. L'entraîneur était alors amené à réaliser un compromis qui consistait à libérer des temps pour se reposer tout en occupant ces temps, pour éviter que les athlètes ne s'ennuient. Des verbatim permettaient d'illustrerce compromis :

«quand on est en stage, c'est vrai que c'est une pression, on se sent obligé de faire de l'animation, c'est très lourd, au moins, là ils se reposent. » escrime

La libération de temps pour les athlètes induisait ici un changement dans les activités des entraîneurs, qui étaient amenés à « occuper » les athlètes au delà des temps d'entraînement.

Par ailleurs, ces temps «libérés » laissaient parfois éclater des tensions et des rivalités entre les athlètes. Des verbatim permettaient de les illustrer :

«le problème des stages c'est qu'il faut occuper les athlètes, sinon ils font les cons, ils s'ennuient [...] ils se toisent un petit peu entre eux [...]. Puis y'a la fatigue, un coup de spleen au bout d'une dizaine de jours et ensuite peuvent arriver les petits incidents, les enguelades. 
Des fois y'a des bagarres, des coups de poing qui partent. Y'a des tensions, des rivalités, certaines sont affichées, d'autres éclatent. » athlétisme

Deux types d'organisation des temps pouvaient alors s'observer: le premier révélait des temps très prégnants où se succédaient des activités au cours de la journée à l'INSEP et le second montrait une augmentation des temps libérés qui induisait une réorganisation des temps pour chacun des agents et des changements dans leurs activités, lors des stages réalisés à l'extérieur de l'INSEP.

L'activité d'organisation des temps dans l'entraînement est apparue comme une activité de compromis, de réduction des tensions entre des composantes en interaction. Ces tensions se découvraient au cours de l'exercice, les compromis étaient réalisés de façon contingente aux problèmes rencontrés.

\subsection{Les organisations temporelles : des inventions locales propres à chaque sport}

Le troisième type de résultats a permis d'identifier des organisations temporelles d'empans variés. Des organisations larges se déclinaient en une juxtaposition d'organisations plus réduites dans le temps, dans lesquelles on pouvait distinguer d'autres organisations d'empan plus réduit. Ces organisations étaient bornées par des événements ou par un temps calendaire. Le processus de génération des organisations temporelles a permis de générer deux types de bornages dans le temps et trois organisations typiques en fonction des sports considérés.

\subsubsection{Deux types de bornage des temps}

L'analyse des organisations temporelles a montré deux types de bornage des temps. L'organisation de la saison est apparue bornée par des événements: les compétitions majeures. Ces événements bornaient des périodes larges. Chaque période large était ensuite organisée en des périodes de plus en plus réduites, bornées par un temps calendaire. Des verbatim ont permis d'illustrer ces bornages différenciés des temps :

«Les championnats de France, c'est en mai, les championnats d'Europe ou les Jeux Olympiques c'est l'été. Y'a trois cycles par saison. Le premier se termine en décembre, avec les championnats d'Europe en petit bassin. Le deuxième cycle se termine avec les championnats de France et le troisième avec la compétition de l'été [...] chaque cycle est découpé en micro-cycles. Le cycle il est simple : on se remet en condition, on fait de la force, on fait de la force endurance, après on a tant de volume par semaine et après j'ai une organisation de semaine. A l'intérieur de chaque semaine, j'ai trois séances de muscul et j'ai tant de séances dans l'eau, et chaque séance a... ». natation

La saison était organisée en trois périodes bornées par les championnats d'Europe en petit bassin, les championnats de France et les championnats d'Europe. Chaque période était ainsi découpée en périodes d'empan de plus en plus réduit. 


\subsubsection{Trois organisations temporelles typiques au cours de la saison}

L'analyse des résultats a révélé différents niveaux d'organisation des temps au cours de la saison : des organisations relativement larges bornées par des compétitions majeures ou par la fin d'un championnat et des organisations plus réduites, enchâssées dans les précédentes et bornées par un temps calendaire. Trois organisations larges et typiques ont été identifiées au cours de la saison; elles consistaient en : (a) une organisation en deux périodes larges pour le groupe, (b) une organisation en trois périodes larges pour le groupe (b) et une organisations en différentes périodes pour chacun des athlètes.

\subsubsection{Une organisation en deux périodes larges pour le groupe}

Cette organisation typique consistait en deux larges périodes bornées par des événements. Elle concernait la lutte, le judo, l'escrime, l'athlétisme, la gymnastique, le basket-ball. Les événements étaient les championnats d'Europe et du Monde en lutte (i.e. «les CE c'est en avril, les championnats du monde c'est en septembre octobre »), en escrime (i.e. «les championnats du monde en octobre et les championnats d'Europe en juillet»), en gymnastique (i.e. "le championnats d'Europe sont au mois d'avril, les championnats du monde sont en octobre novembre »), en judo (i.e. "les championnats d'Europe sont en avril et les championnats du monde sont en septembre »). Les événements étaient : les championnats d'Europe et de France en basket-ball (i.e. «la saison est bornée par le championnat d'Europe qui se situe en juillet août. On a une saison hivernale qui va s'arrêter un mois avant la préparation aux Europe, elle a commencé aux alentours du 28 août et elle s'arrête aux alentours du 15 mai. »). Les événements concernaient les compétitions hivernales et estivales en athlétisme (i.e. "y'a une phase de compétition hivernale et une phase de compétitions estivales qui se termine avec les championnats du Monde. »).

Les périodes présentaient des durées proches, sauf en basket-ball où une période estivale courte succédait à une période « hivernale » longue et en escrime où la durée entre les championnats d'Europe et du Monde était plus courte que la durée entre les championnats du Monde et d'Europe. Ces périodes concernaient le groupe d'entraînement, même si tous les athlètes ne participaient pas aux événements bornant la période.

A chaque période large, se superposaient des périodes plus courtes, bornées par un temps calendaire. Les verbatim ont permis de l'illustrer :

«la $1^{\grave{r} r}$ période dure trois semaines: rentrée en août septembre, travail foncier, travail d'oxygénation, travail d'endurance spécifique où on développe la forme athlétique des gym et leur capacité à développer des charges élevées d'entraînement. 2è période, on va rentrer dans un domaine plus technique, les premières compet arrivent en novembre. Dès le mois d'octobre, on rentre dans une période de mouvements complets, puis on va essayer de maintenir ce travail jusqu'aux championnats du monde. Ensuite on maintient le niveau compétitif parce qu'il y a des tournois en pagaille. Les tournois s'arrêtent mi-décembre, ils repartent dans leur famille pour 15 jours. Janvier février on reprend sur le même rythme que août septembre, pour qu'ils soient très vite opérationnels au mois de mars, puis on maintient jusqu'au championnats d'Europe. » gymnastique

La première période présente une phase de reprise, une phase de travail technique, une phase de mouvements complets, une phase de maintien des mouvements complets. Chaque sous période fait ensuite l'objet d'une programmation spécifique : 
«j'ai un programme par semaine. Je fais des programmes sur quatre semaines et après je rectifie. Je fais un programme par athlète du lundi au samedi et je le donne à chaque athlète. Le matin on fait préparation physique et un ou deux agrès et l'après-midi, on fait le travail d'éléments sur les quatre agrès. Tous les mercredi matin, on fait un tour complet des finales par appareil, y'a les sorties, on règle les problèmes de petites chutes. Le jeudi matin, c'est la répétition du test [...] le programme est fait de compétition en compétition. Il est fait par rapport au programme des compétitions ».

D'une organisation préalable pour le groupe, on est passé progressivement à une organisation pour chacun des athlètes. Cette individualisation s'observait sur des périodes courtes. Quelquefois, la finalité et le contenu de ces périodes courtes étaient repris sur la période suivante, montrant par là une imbrication entre les périodes successives. Des verbatim ont permis de l'illustrer :

«le planning hebdomadaire est valable sur trois semaines. C'est écrit le jour de notre entretien et les heures. A l'issue, du cycle, on peut décider de le reproduire ou non.» athlétisme

\subsubsection{Une organisation en trois périodes larges pour le groupe}

Le deuxième type d'organisation de la saison révélait trois périodes larges pour le groupe d'entraînement. Ces périodes étaient bornées par des événements importants ou majeurs. Cette organisation était typique en natation. Les événements étaient: les championnats d'Europe en petit bassin, les championnats de France et les championnats d'Europe ou du monde selon les années. Chaque événement bornait une période, appelée cycle, qui était ensuite à l'origine de périodes de plus en plus petites :

«y'a trois cycles par saison. Le premier se termine en décembre avec les championnats d'Europe en petit bassin. Le deuxième cycle se termine avec les championnats de France et le troisième avec la compétition de l'été [...] chaque cycle est découpé en micro-cycle. Le cycle il est simple: on se remet en condition, on fait de la force, on fait de la force endurance, après on a tant de volume par semaine et après j'ai une organisation de semaine. A l'intérieur de chaque semaine, j'ai trois séances de muscul et j'ai tant de séances dans l'eau, et chaque séance a...»

Les organisations globales étaient destinées au groupe d'entraînement. Les organisations locales étaient alors précisées pour chacun des athlètes, à travers le contenu d'entraînement.

\subsubsection{Une organisation en différentes périodes pour chacun des athlètes}

Le troisième type d'organisation révélait une succession de périodes pour chacun des athlètes au cours de la saison. Cette organisation «à la carte » est apparue en badminton et en cyclisme. En badminton, la saison était rythmée par la succession régulière des compétitions. Un état de forme optimal devait être maintenu tout au long de l'année ; peu de périodes de repos total étaient prévues. Les périodes se succédaient tout en s'imbriquant les unes dans les autres. Des verbatim permettaient de l'illustrer :

"Elle fait seize à dix-sept compétitions par an et quasiment toutes les compétitions majeures. On fixe des périodes de quinze jours, on se fixe des objectifs en fonction du dernier tournoi. 
On fait un bilan, on voit si on continue tel domaine technique ou tactique ou si on change [...].elle s'arrête presque jamais. »

Les périodes se succédaient tout en s'imbriquant les unes dans les autres.

En cyclisme, les organisations temporelles étaient déterminées en début d'année pour chaque athlète, en fonction de sa participation aux différentes compétitions :

«au mois de septembre, on nomme les athlètes qui vont participer aux différents objectifs [...] ils vont avoir des cycles différents selon les objectifs pour lesquels ils sont inscrits [...] on a des cycles de trois semaines à l'issue desquels ils doivent être capables de faire un nombre de répétitions avec un braquet. Je leur donne un programme par semaine. »

Ce type d'organisation était d'emblée individualisé. Il était induit par le mode d'organisation des compétitions à travers lequel les inscriptions étaient très tôt nominatives.

\subsubsection{Des relations particulières entre les périodes}

L'analyse des organisations générées a permis d'identifier des relations particulières entre les périodes identifiées. Ainsi des relations de juxtaposition sont apparues entre les périodes larges.

Des relations de superposition ont été mises en évidence. Les périodes larges bornées par les événements majeurs se superposaient à des périodes plus petites finalisées par un état de préparation particulier, qui se superposaient elles-mêmes à des périodes encore plus courtes finalisées par des apprentissages spécifiques, des ajustements, des enchaînements.

Des relations d'imbrication ont été révélées entre les différentes organisations générées. En effet, chaque période prenait appui sur la précédente qu'elle poursuivait (e.g. «les temps d'apprentissage c'est tout le temps, hormis les périodes de compétition » judo). La succession des périodes induisait l'atteinte des objectifs de l'une pour s'acheminer vers l'autre. En gymnastique, la deuxième période visait à maintenir le travail réalisé en première période : la deuxième période poursuivait la première, de laquelle elle ne pouvait être isolée. Les organisations temporelles identifiées ci-dessus concernaient l'entraînement. Ces organisations, ces cadres temporels étaient associés à d'autres cadres liés aux activités de formation, d'activité professionnelle, au repos...

Des relations de concurrence apparaissaient entre les cadres destinés aux différentes activités. Des relations de concurrence sont apparues entre des cadres centrés sur l'entraînement et des cadres centrés sur les activités scolaires :

«à certains moments, les athlètes passent le bac, donc ils sont pris » gymnastique

Une autre concurrence est apparue en escrime entre les cadres temporels liés à l'activité professionnelle de certains athlètes et ceux inhérents aux activités d'entraînement et prévus par l'institution :

« des deux athlètes qu'on prend actuellement à 9h, l'un est en convention, donc il prend sa leçon, il se douche, il va bosser jusqu'à la fin de la journée, l'autre c'est une convention de stage. Les autres athlètes s'entraînent à partir de 10 ou 11 h selon l'heure de fin de cours ». 
3.3.4. Une confrontation entre les temps prévus et les temps réels

Cette organisation des temps prévus ou anticipés se confrontait à des temps réels (ou temps des événements) qui permettaient ou non de réaliser «ce qui était prévu ». Ces temps réels étaient souvent source d'événements imprévus qui amenaient à renouveler rapidement l'organisation initiale. Des verbatim ont permis de l'illustrer :

«fin novembre, elle a fait des performances d'entraînement très impressionnantes pour la saison. Elle a eu un premier problème à l'épaule et nous avons stoppé l'entraînement physique, puis ça s'est renouvelé. Chaque fois nous n'avons fait que reprendre l'entraînement sous forme d'éducatifs » athlétisme

«Avant les Jeux [..., l'athlète] ne mettait plus un pied devant l'autre. On était en stage et on était obligé de tricher sur les temps... Je dormais très mal. Les autres coureurs ne comprenaient pas, ils disaient: on est obligé de freiner dans sa roue [...] pourquoi... l'ennui [...] et il est reparti » cyclisme

Ce renouvellement de l'organisation initiale était finalisé par la préservation de l'intégrité physique, par le maintien d'un état de forme et de préparation psychologique des athlètes.

L'organisation initiale était quelquefois modifiée du fait d'imprévus liés aux compétitions, aux examens, aux réunions inopinées des entraîneurs :

«Ca peut être une compétition imprévue, un organisateur privé qui veut absolument avoir les athlètes et qui prévient à six jours. Ben il y va et y'a pas de problème. » cyclisme

«j'avais inscrit l'athlète à trois compétitions successives. On a toujours les tableaux une semaine à l'avance et quand on était en Chine, on s'est aperçu qu'elle n'était pas dans le tableau de Taïwan, ils avaient oublié de l'inscrire. » badminton

«les imprévus peuvent être un problème de boulot ou d'examen. Les partiels de cette semaine, je les ai eus la semaine dernière ». escrime

«t'as toujours des réunions qui viennent se greffer » judo

Ces imprévus amenaient les entraîneurs à réaliser des compromis entre ce qui était prévu et les circonstances de mise en œuvre locales. Le caractère imprévisible de ces événements concernait davantage le moment d'occurrence de l'événement que la nature de l'événement (mis à part le problème lié à la non inscription en tournoi). Les entraîneurs, avec l'expérience, semblaient se préparer à ces «imprévus» qui finalement apparaissaient «prévisibles ».

L'organisation du temps dans les activités d'entraînement est apparue co-générée par deux types de temps : un temps linéaire et un temps non linéaire. Le temps linéaire renvoyait : (a) aux calendriers communiqués par les fédérations internationales et nationales, (b) aux temps de l'institution qui contribuaient eux-mêmes à organiser le temps des différentes activités des acteurs, et (c) aux connaissances liées au processus d'entraînement. Les temps calendaires apparaissaient ponctués de dates, qui correspondaient à des compétitions d'importance variée aux plans international et national. Le caractère continu du temps était mis en évidence à travers la succession de dates correspondant au déroulement du temps au 
cours de la saison. Les temps de l'institution étaient eux-mêmes déterminés par les temps scolaires ou universitaires qui rythmaient l'alternance de périodes de travail et de repos. Les temps discontinus renvoyaient quant à eux, à la contingence des actions, aux projets poursuivis et aux événements actuels. 


\section{Discussion}

Les résultats ont montré que l'organisation des temps dans les activités d'entraînement consistait en des inventions locales dans un cadre contraignant. L'organisation des temps dans les activités d'entraînement est apparue générée par un processus circulaire, qui mobilisait trois paramètres en interaction : les compétitions, les projets et l'entraînement ainsi que leurs composantes. Le fonctionnement du processus dans un cadre local et contraignant était à l'origine de tensions, de concurrences, de résistances entre les composantes des paramètres. L'entrâneur était amené à trouver des compromis pour réduire ces résistances et concurrences. Trois organisations temporelles typiques ont été identifiées au cours de la saison : une organisation en deux périodes pour le groupe, une organisation en trois périodes pour le groupe et une organisation en différentes périodes pour chacun des athlètes. Ces organisations se découpaient en des organisations plus réduites; elles présentaient des relations particulières. Les résultats sont discutés en trois temps : (a) un processus circulaire de génération des organisations temporelles s'appuyant sur une vision holistique des activités d'entraînement, (b) des inventions locales finalisées par la réussite et, (c) des organisations temporelles typiques liées à des temporalités variées. Des perspectives pour la formation sont ensuite envisagées.

\subsection{Un processus circulaire de génération des organisations temporelles révélant une vision holistique des activités d'entraînement}

Les résultats ont indiqué que l'organisation du temps dans les activités d'entraînement s'appuyait sur un processus circulaire, amenant à anticiper l'avenir, à revenir sur le passé pour se projeter dans l'avenir. Ce processus s'appuie sur la conception du temps du projet proposée par Dupuy (2002). Le futur est tenu pour fixe. Une anticipation particulière de l'avenir a des conséquences sur le passé qui l'anticipe et qui réagit en retour. Le temps représente une boucle dans laquelle le passé et l'avenir se déterminent réciproquement. Dans cette étude, la saison était organisée à partir d'une anticipation des compétitions majeures et d'un retour vers le passé. L'anticipation portait à la fois sur les dates des compétitions, sur leur enjeu, sur les résultats et sur l'état de forme attendus des athlètes. Le retour vers le passé et la projection dans l'avenir s'appuyaient sur l'interaction de trois paramètres: les compétitions, les projets et l'entraînement. Chaque paramètre présentait un ensemble de composantes. Le paramètre lié à la compétition contenait des composantes inhérentes : (a) aux échéances compétitives, (b) au type de compétition, (c) au programme de la compétition. Le paramètre lié aux projets consistait en des composantes liées : (a) aux projets des institutions : la mise en œuvre de conditions optimales pour organiser la vie des athlètes, l'obtention de résultats sportifs et la gestion d'un budget pour le groupe d'entraînement, et (b) aux projets des agents : les résultats sportifs attendus, la disponibilité des athlètes, et les gains financiers attendus par les athlètes. Le paramètre lié à l'entrainement contenait des composantes inhérentes : (a) au temps requis pour préparer les échéances et pour se reposer, (b) à l'augmentation progressive de la charge d'entraînement, (c) à la détermination de temps pour réaliser un travail ou un effort particulier et (d) à l'espace et à la sécurité.

Ce processus d'organisation du temps différait en partie du processus utilisé dans les planifications issues de la littérature sur l'entraînement (e.g., Platonov, 1988 ; Weineck, 1986 ; Pradet, 1996). Il ne se réduisait pas à la mobilisation de connaissances génériques sur l'athlète à l'entraînement mais il s'appuyait sur une vision holistique des activités 
d'entraînement. A travers cette vision, les agents étaient engagés à la fois dans un processus de production de performance mais aussi dans des projets liés aux études, à un métier, à une famille, à des gains financiers. Ils évoluaient dans une institution particulière : l'INSEP, à laquelle était rattaché un pôle qui dépendait également d'une autre institution, la fédération sportive concernée. Cette prise en compte des projets imbriqués des agents et des institutions apparaissaient peu dans les travaux de Salmela (1996). Les temps considérés dans l'étude dépassaient les seuls temps de l'entraînement et de la compétition pris en compte par la littérature sur l'entraînement. Dans cette littérature, les athlètes apparaissent génériques : leurs aptitudes, leurs motivations et leurs buts sportifs doivent être spécifiés par le planificateur. Dans l'étude actuelle et dans les études de Saury \& Durand (1997) et de Sève \& Durand (1999), les athlètes étaient singuliers. Les organisations temporelles apparaissaient contingentes aux actions des agents, aux événements anticipés et aux événements réels. Au delà des aspects sportifs, mécaniques, psychologiques et physiologiques, les organisations temporelles étaient générées à partir de composantes liées à l'être social, à son histoire et à son avenir; elles étaient singulières. Du domaine de la généralisation, on est passé au domaine de la singularisation. Du domaine de la prescription, on est passé au domaine du « réalisé » de «ce qui se fait ». Les temps révélés dans l'analyse étaient générés par un processus circulaire, mobilisant trois paramètres en interaction : les compétitions, les projets et l'entraînement. En référence aux travaux de Le Moigne (1998), les organisations temporelles ainsi générées sont apparues téléologiques : les temps finalisaient l'activité et étaient finalisés par l'activité. La récursivité s'observait au niveau des objectifs concomitant au bornage de la période (e.g., atteindre le niveau de forme optimal) et de l'activité permettant d'atteindre ces objectifs (e.g., mise en place des conditions pour atteindre ce niveau de forme optimal).

\subsection{Les organisations temporelles : des inventions locales finalisées par la réussite sportive dans un cadre contraignant}

L'analyse des interactions entre les composantes des paramètres a indiqué des résistances, des concurrences qui amenaient à la réalisation de compromis. Ainsi l'entraîneur de lutte rapportait :

« elle voudrait faire 15 jours de préparation pour aller aux championnats d'Europe [alors qu'] il faut au minimum deux tournois, plus trois semaines ou plutôt un mois de préparation [...] ce n'est pas du tout la même approche que si elle est célibataire, jeune et qu'elle est au pôle [...] je suis conscient que ça leur en demande plus et je dois donc aménager. Y'a une négociation par rapport à l'objectif »).

Ces résistances concernaient deux composantes liées aux projets des agents (résultats sportifs attendus et vie au delà de l'entraînement) et au temps requis pour préparer le type de compétition. L'entraîneur était amené à transformer le problème en fonction des résistances observées entre les composantes. D'un problème de détermination d'une durée optimale pour préparer une compétition majeure, l'entraîneur faisait face à un problème d'adéquation entre la détermination de cette durée optimale et le temps consenti par l'athlète pour réaliser cette préparation. La résistance des composantes le conduisait à inventer de nouveaux cadres, de nouvelles organisations temporelles. Cette activité de compromis, fondée sur une transformation du problème à résoudre a été analysée par Jeannot (2005), dans une étude sur les métiers flous. L'auteur a montré que l'activité de compromis s'accompagnait d'une transformation continue des données du problème en fonction des résistances rencontrées. Dans l'étude actuelle, la résistance des composantes conduisait l'entraîneur à inventer de 
nouveaux cadres, de nouvelles organisations temporelles. Elle l'amenait également à anticiper l'avenir ; cette anticipation rétroagissait sur son action immédiate. Ainsi, l'entraîneur de lutte anticipait un niveau de préparation optimale pour l'athlète en vue des Championnats d'Europe. Cette anticipation qui incluait également la possibilité de s'entraîner de son côté (i.e. "sachant qu'elle va s'entraîner de son côté »), rétroagissait sur l'action de détermination de la durée de la période de préparation avec le groupe France. Parfois, la recherche de compromis était abandonnée, car elle aurait induit un éloignement important de l'objectif majeur : l'obtention de résultats en compétition. Ainsi en escrime, la durée optimale de la période de préparation à une compétition était parfois tronquée afin de permettre de participer à une compétition importante :

«J'essaie de choisir des épreuves qu'on puisse préparer correctement, à savoir, il me faut au moins quatre semaines avant une épreuve importante que je vais considérer comme sélective [...] il y a souvent des couacs : fin janvier début février j'avais deux épreuves très très importantes, j'avais ce qu'on appelle les grands prix, on est sûr qu'il y a beaucoup de monde, je les ai pris comme épreuves sélectives, c'était les seules, donc j'avais vraiment pas le choix. »

Les résultats ont également montré que les agents déployaient des solutions locales afin de mettre en place les conditions de la réussite sportive. Dans le cadre de la lutte, deux organisations co-existaient : une organisation pour les athlètes célibataires vivant au pôle, et une organisation pour quelques athlètes mariées vivant en province. Lors des stages de regroupement, ces organisations étaient combinées. Ces solutions locales, basées sur des négociations entre les personnes sont consistantes avec celles présentées par Duc (1993), sur le travail de chantier. L'organisation et la réalisation du travail exige des inventivités locales pour s'adapter aux réalités souvent imprévisibles des événements et des actions de chacun. Le travail présente une prescription floue : les agents savent globalement ce qu'ils doivent faire, mais ne savent ni comment, ni quand, ni avec qui ils vont effectivement le faire. L'organisation du temps dans les activités d'entraînement pourrait être assimilée à un travail à prescription floue: l'organisation des temps est finalisée par la réussite aux différentes compétitions, tout en étant largement indéterminée au préalable. Cette indétermination concerne les projets des agents et les compétitions. L'indétermination concerne le programme au cours de la saison. Ce programme est peu défini, il apparaît défini par des injonctions concernant la finalité (e.g. "maintenir nos cinq médailles aux championnats d'Europe », lutte). Sa détermination est laissée à la charge des entraîneurs. En référence aux travaux de Jeannot (2001), l'indétermination semble située en amont du travail d'organisation. L'organisation est à construire dans un contexte local. Cette indétermination située en amont du travail révélé dans cette étude se distingue d'une indétermination située strictement en aval du travail. Cette dernière renvoie aux planifications et aux régulations conçues dans la littérature sur l'entrainement. Le travail consiste alors à appliquer des connaissances, des savoirs formels dans des situations concrètes. De l'application de règles et de leur ajustement dans un contexte largement prédéterminé par des connaissances souvent génériques, on est passé à la détermination sans cesse actualisée d'un contexte local, à partir de connaissances non seulement génériques (e.g. «il faut un mois de préparation convenable »), mais aussi expérientielles et phénoménologiques («ce n'est pas du tout la même approche que si elle est célibataire, jeune et qu'elle est au pôle »). En référence à Jeannot (2001), on pourrait associer le travail du temps à une confrontation à ce qui résiste. L'activité de compromis résulte de cette confrontation. 


\subsection{Des organisations temporelles typiques liées à des temporalités variées}

Deux types d'organisations temporelles ont été générées : le premier concernait la saison, il était borné par un temps événementiel : les compétitions majeures. Le second type, enchâssé dans le précédent était borné par un temps calendaire : les cycles de trois à quatre semaines. Chaque organisation pouvait être assimilée à un cadre temporel, en référence aux travaux de Grossin (1996) et à ceux de Valax (1986). Quel que soit le bornage du temps, chaque cadre définissait une période (e.g., "il faut un mois de préparation, en sachant qu'il y a une période de préparation antérieure[...] après les Europe, il y a une phase de quinze jours de repos et puis derrière on va reprendre l'entraînement une fois par jour »). Ces périodes pouvaient être relativement courtes, comme celles su citées ou plus longues (e.g. "C'est deux échéances chaque année, les championnats d'Europe c'est en avril, les championnats du monde c'est en septembre, octobre »). Les périodes courtes se succédaient dans le temps, elles étaient enchâssées dans des périodes plus longues qui se succédaient également. L'analyse a montré que certains cadres étaient imbriqués dans d'autres, le contenu des périodes présentait alors de fortes similitudes (e.g., «les temps d'apprentissage c'est tout le temps, hormis les périodes de compétition » judo). Certains cadres se chevauchaient sur d'autres, créant ainsi des tensions, des résistances entre les cadres, qui étaient à l'origine d'un ajustement dans l'organisation (e.g., «à $9 h$ [...] il prend sa leçon, il se douche il va bosser jusqu'à la fin de la journée[...] les autres athlètes s'entraînent à partir de $10 \mathrm{~h} »)$. Ces relations entre les cadres temporels finalisés par l'entraînement ou par d'autres activités sont consistantes avec les travaux de Grossin (1996).

Les modes de structuration des temps dans les activités d'entraînement ont révélé deux types de temporalité : une temporalité longue qui renvoyait aux projets, au long terme et une temporalité courte qui s'appuyait sur l'action immédiate, sur les événements, sur le court terme. La temporalité longue consistait en l'organisation des temps au cours de la saison et la temporalité courte se référait à l'activité de compromis, de renouvellement des organisations temporelles face aux imprévus, à l'urgence. Ces temporalités longues sont consistantes avec les deux types de temps distingués par Bouquet (2004), dans une étude sur l'organisation du temps dans une maison spécialisée. Ces deux types de étaient le temps circulaire et le temps singulier. Ils sont également consistants avec les notions de temps indifférencié et de temps pour faire face à l'urgence, soulignés par Yanda Ngary (2006) dans une étude sur l'évolution des activités agricoles. Le temps circulaire s'inscrit dans la répétition d'événements précis, significatifs, qui permettent de se repérer. Le temps singulier ou temps de l'urgence est un temps événementiel qui ne peut être prévu. Il est contingent, il réagit aux circonstances locales. Dans l'étude, le temps circulaire renvoyait à la fois à la succession des séances d'entraînement, des compétitions, des stages, et à la succession des temps destinés aux différentes activités de la journée organisées au sein de l'INSEP (formation, repos, alimentation). Le temps singulier était celui de l'urgence, des imprévus. Ce temps relevait d'interventions singulières, de solutions locales (e.g., suite à une blessure répétée, «nous n'avons fait que reprendre l'entraînement à partir d'éducatifs »). Ces temporalités longues ou courtes sont consistantes avec les niveaux macro et micro envisagés par Dubar (2004). Un troisième niveau, le niveau méso, s'insère entre les deux autres. Il croise à la fois des temporalités institutionnelles ou sociales et des temporalités événementielles ou biographiques. Le niveau méso est un temps de la structure, des contraintes de production. Des temporalités s'enchâssent et s'imbriquent dans les mondes sociaux du travail et du « hors travail ». C'est dans ce niveau méso que se construisent les temps dans les activités d'entraînement. Cette construction était générée par un processus circulaire qui s'appuyait sur trois paramètres et sur treize composantes en interaction. Ainsi, L'entraîneur était amené à prendre en compte les exigences de résultats de la fédération (e.g., «avoir cinq médailles aux 
championnats d'Europe »), le budget alloué (e.g., "y'a des coupes sombres s'il n'a pas obtenu le budget qu'il voulait») et un ensemble d'autres composantes. A ce niveau méso apparaissaient également des tensions, des résistances, des rivalités entre les composantes en interaction. Ces temps de l'urgence ont été relativement peu mis en évidence dans cette étude, qui s'est centrée sur des temps plus longs. Ce constat constitue une limite à cette étude. Pour appréhender ce temps de l'urgence, il apparaît intéressant de suivre les agents dans leurs activités quotidiennes, afin de mieux comprendre les tensions, les résistances qui émergent de l'activité en cours, ainsi que les compromis, les adaptations, les ajustements réalisés dans l'urgence. Ce type d'étude permettrait de mettre en évidence les adaptations permanentes relevant souvent d'un «bricolage cognitif » réalisé dans la dynamique des situations. Il pourrait faire l'objet d'une prochaine étude.

\subsection{Des perspectives pour la formation des entraîneurs}

Cette étude présente des perspectives à la fois pour les activités d'entraînement et de formation. Elle amène à réinterroger l'organisation des temps, en prenant en compte le contexte à la fois singulier et dynamique des activités d'entraînement. Elle conduit à une prise de recul sur les éléments considérés dans le processus d'organisation des temps, ne se limitant pas à la principale prise en compte de connaissances scientifiques sur l'entraînement. Elle amène à réinterroger l'organisation des temps, en prenant en compte le contexte à la fois singulier et dynamique des activités d'entraînement. Elle incite à adopter une vision holistique et située des activités d'entraînement, incluant les agents, les institutions, les compétitions distinguées selon leurs échéances, les enjeux qu'elles représentent, et le programme qui leur est associé. Elle insiste sur le caractère local des organisations temporelles générées et sur leur caractère en partie instable. Elle amène ainsi à changer de regard sur les activités d'entraînement. Celles-ci n'apparaissent plus comme une stricte application de connaissances développées par d'autres, mais plutôt comme une activité chaotique, une activité de compromis et d'inventions locales finalisées par les réussites des agents et réalisées dans un cadre contraignant.

La formation, lorsqu'elle est envisagée sous la forme d'un enseignement disciplinaire, semble rendre plus difficile les conditions d'une approche holistique des activités d'entraînement. L'entraîneur en formation ou le futur entraîneur envisage le temps le plus souvent sous des aspects disciplinaires. Le temps est alors organisé en fonction de progressions au plan de la sollicitation de processus énergétiques finalisée par la production d'un effort particulier, ou de l'apprentissage de nouveaux gestes, de nouvelles techniques, ou tactiques, ou l'amélioration des points forts et la réduction des points faibles. La formation s'appuie alors sur un modèle idéal, explicatif et plutôt prescriptif. Dans leur activité professionnelle, les entraîneurs réalisent des compromis. Ces derniers sont rendus possibles par une indispensable connaissance du contexte local de l'entraînement, considéré dans des espaces temps variés, avec des agents qui peuvent être différents. Cette démarche de compromis pourrait être envisagée davantage dans le cadre de la formation à travers une approche thématique, qui amène les agents à coordonner les connaissances issues des différentes disciplines et les connaissances issues de l'expérience des agents et de l'analyse du contexte. Cette formation pourrait être organisée sous forme d'études de cas liées à des organisations temporelles identifiées et / ou de conceptions d'organisations temporelles. 


\section{Conclusion}

Cette étude visait à comprendre l'organisation des temps dans les activités d'entraînement. Les organisations temporelles étaient générées par un processus circulaire qui s'appuyait sur trois paramètres et treize composantes en interaction. Les interactions entre les paramètres et leurs composantes ont révélé des tensions, des concurrences, qui conduisaient le plus souvent à la réalisation de compromis, finalisée par une recherche d'optimum dans la préparation des athlètes aux compétitions. Les temps ainsi identifiés sont apparus multiples, discontinus, chaotiques, faisant l'objet de réorganisations successives, d'adaptations locales et dynamiques aux contextes. L'analyse des organisations temporelles a montré des relations de superposition, de succession, d'imbrication. Les temps ainsi révélés étaient issus de compromis entre des éléments contextuels et dynamiques et des connaissances génériques.

Cette étude présente des perspectives à la fois pour les activités d'entraînement et de formation. Elle amène à réinterroger l'organisation des temps, en prenant en compte le contexte à la fois singulier et dynamique des activités d'entraînement. Elle conduit à une prise de recul sur les éléments considérés dans le processus d'organisation des temps, ne se limitant pas à la principale prise en compte de connaissances génériques sur l'entraînement. Elle incite à adopter une vision holistique et située des activités d'entraînement, incluant les agents, les institutions, les compétitions distinguées selon leurs échéances, les enjeux qu'elles représentent, et le programme qui leur est associé. Elle insiste sur le caractère local des organisations temporelles et sur leur caractère en partie instable. L'organisation des temps apparaît chaotique, elle est issue de compromis et d'inventions locales finalisées par les réussites des agents et réalisées dans un cadre contraignant. 


\section{Références}

Audigier, F.(2007). Temps subis, temps construits. In D. Lahanier-Reuter, \& E. Roditi (Eds), Questions de temporalités (pp. 15-27). Villeneuve d'Asq: Presses universitaires du Septentrion.

Bachelard, G. (1936). La dialectique de la durée. Paris : PUF.

Bergson, H. (1981, 154è édition, 1ère édition 1907). L'évolution créatrice. Paris: PUF.

Bouchetal Pellegri, F., Leseur, V., \& Debois, N. (2007). Carrière sportive - Projet de vie. Paris : Editions INSEP.

Corbin, J., \& Strauss, A. (1990). Basics of qualitative research: Grounded theory. New-Bury Park, CA: Sage.

Bouquet, B. (2004). Les temps des l'intervention sociale. In collectif Griot, Figures du temps (pp. 221-241). Paris : L'Harmattan.

Discours de Mme la Ministre de la Santé, de la Jeunesse et des Sports : téléchargé le 25 octobre 2007, www.jeunesse-sports.gouv.fr/accueil_5_ministre_201/discours_220/ discours-insep-vincennes_1629.html

Dubar, C. (2004). Régimes de temporalités et mutation des temps sociaux. Temporalités, 1 , 100-119.

Duc, M. (1993). Le travail en chantier. Toulouse : Octarès.

Dupuy, J.-P. (2002). Pour un catastrophisme éclairé. Paris : Seuil.

Grossin, W. (1996). Pour une science des temps : introduction à l'écologie temporelle. Toulouse : Octarès.

Jeannot, G. (2000). Faire du général avec du singulier: les chefs de service d'une DDE et l'aménagement, Les annales de la recherche urbaine, 88, 49-57.

Jeannot, G. (2001). Le travail public : un exemple dans le développement local. In G. Jeannot, \& P. Veltz, Le travail entre l'entreprise et la cité (pp. 125-132). Editions de l'Aube.

Jeannot, G. (2005). Les métiers flous. Travail et action publique. Toulouse : Octarès.

Kirshner, D., \& Whitson, J.A. (Eds). Situated cognition: Social, semiotic and psychological perspectives. Mahwah: Lawrence Erlbaum associates.

Le Moigne, J.-L. (1998). Temps du travail et travail du temps : «on ne peut pas ne pas changer $»$. www.ergonomie-self.org/actes/SELF98.pdf

Mignon, P. (2007). Laure et Philippe, ou les psychodrames du sportif entrepreneur. Revue Esprit, août-septembre, 38-54. 
Ministère de la Santé de la jeunesse et des sports (mis à jour le 12 juillet 2007). Les dispositifs d'aide aux sportifs de haut niveau. http://www.jeunesse-sports.gouv.fr/sports_1/sporthaut-niveau-sport-professionnel_69/sport-haut-niveau_373/les-dispositifs-aides-sportifve-haut-niveau 590.html

Platonov, V.N. (1988). L'entraînement sportif. Théorie et méthodologie. Paris : Revue EPS.

Pradet, M. (1996). La préparation physique. Paris : Editions INSEP.

Salmela, J. H (1996). Great job coach! Getting the edge from proven winners. Ottawa: Potentium.

Saury, J., Durand, M., \& Theureau, J. (1997). L'action d'un entraîneur expert en voile en situation de compétition : étude de cas. Contribution à une analyse ergonomique de l'entraînement. Science et Motricité, 21, 21-35.

Schön, D. A. (1983). The reflexive practionner. New York : Basic Books.

Sève, C. \& Durand, M. (1999). L'action de l'entraîneur de tennis de table comme action située, Avante, 5, 1, 69-85.

Suchman, L. (1987). Plans and situated action. Cambridge : University Press.

Valax, M.-F. (1986). Cadre temporel et planification des tâches quotidiennes : Etude de la structure des plans journaliers chez les agriculteurs, Le Travail Humain, 49, 4, 371-374.

Weineck, J. (1986). Manuel d'entraînement. Paris : Vigot

Yanda Ngary, B. (2006). Du temps des activités agricoles au temps des activités commerciales. In J. Thoemmes, \& G. De Tersac (Eds), Les temporalités sociales : repères méthodologiques (pp. 149-161). Toulouse : Octarès. 


\section{Résumé}

Cette étude est centrée sur l'organisation des temps dans les activités d'entraînement. Dans la littérature sur l'entraînement, l'organisation du temps renvoie plus spécifiquement à une activité de planification et de régulation de temps continus liés à l'entraînement. Les planifications y apparaissent rationnelles. Or l'observation des activités d'entraînement dans des institutions particulières révèle l'existence d'organisations locales et circonstancielles de temps discontinus. Ces derniers renvoient aux différentes activités que les agents déploient dans des espaces variés (e.g., formation, exercice d'un métier). Cette observation amène donc à s'interroger sur des organisations temporelles réduites aux temps de l'entraînement. Cette étude visait à comprendre et à expliquer l'organisation des temps dans les activités d'entraînement, dans un cadre contraignant.

Dix entraîneurs masculins experts de l'INSEP ont participé volontairement à l'étude. Des entretiens ont été menés à partir de traces de l'activité. Les entretiens ont été retranscrits verbatim. L'analyse inductive et déductive a révélé trois types de résultats.

Le premier a montré que les organisations temporelles étaient générées par un processus circulaire. Il consistait à anticiper un niveau de performance pour chaque athlète, lors d'une compétition particulière, et à organiser les temps dans les activités d'entraînement en vue de réaliser cette prédiction. Ce processus s'appuyait sur des éléments locaux propres aux agents, aux institutions, aux événements, aux durées et aux façons envisagées pour réaliser un travail. Ces éléments renvoyaient à trois paramètres en interaction: (a) les compétitions, (b) les projets des agents et ceux des institutions incluant et dépassant le sport et (c) l'entraînement.

Le deuxième type de résultats a mis en évidence des tensions et des résistances entre les éléments. Celles-ci étaient à l'origine de compromis et d'inventions locales dans l'organisation des temps. Les compromis étaient finalisés par les résultats sportifs. Les compromis apparaissaient dynamiques, locaux et circonstanciels.

Le troisième type de résultats a montré trois sortes d'organisations temporelles, selon les disciplines : (a) deux périodes larges pour le groupe, (b) trois périodes larges pour le groupe, et (c) différentes périodes individualisées. Les relations entre les périodes ont été analysées.

Les résultats sont discutés au regard de la littérature sur l'entraînement et la psychologie du sport, sur l'ergonomie cognitive et la sociologie. Des implications pour l'entraînement et la formation sont proposées.

\section{Mots clés}

Activités d'entraînement - Organisations temporelles - inventions locales - structure institutionnelle. 